\title{
Ratios of dijet production cross sections as a function of the absolute difference in rapidity between jets in proton-proton collisions at $\sqrt{s}=7 \mathrm{TeV}$
}

\author{
The CMS Collaboration* \\ CERN, Geneva, Switzerland
}

Received: 3 April 2012 / Revised: 22 October 2012 / Published online: 16 November 2012

(C) CERN for the benefit of the CMS collaboration 2012. This article is published with open access at Springerlink.com

\begin{abstract}
A study of dijet production in proton-proton collisions was performed at $\sqrt{s}=7 \mathrm{TeV}$ for jets with $p_{\mathrm{T}}>$ $35 \mathrm{GeV}$ and $|y|<4.7$ using data collected with the CMS detector at the LHC in 2010. Events with at least one pair of jets are denoted as "inclusive". Events with exactly one pair of jets are called "exclusive". The ratio of the cross section of all pairwise combinations of jets to the exclusive dijet cross section as a function of the rapidity difference between jets $|\Delta y|$ is measured for the first time up to $|\Delta y|=9.2$. The ratio of the cross section for the pair consisting of the most forward and the most backward jet from the inclusive sample to the exclusive dijet cross section is also presented. The predictions of the Monte Carlo event generators PYTHIA6 and PYTHIA8 agree with the measurements. In both ratios the HERWIG++ generator exhibits a more pronounced rise versus $|\Delta y|$ than observed in the data. The BFKL-motivated generators CASCADE and HEJ+ARIADNE predict for these ratios a significantly stronger rise than observed.
\end{abstract}

The measurement of inclusive jet production in $p p$ collisions provides an important testing ground for the Standard Model. Inclusive jet production is well described at LHC energies, over a wide range in jet transverse momentum and rapidity $[1,2]$, by calculations at next-to-leading-order (NLO) in perturbative quantum chromodynamics (QCD) using the Dokshitzer-Gribov-Lipatov-Altarelli-Parisi (DGLAP) approach [3-7] and collinear factorization. The rapidity $y$ is defined as $y=(1 / 2) \log \left[\left(E+p_{z}\right) /\left(E-p_{z}\right)\right]$, where $E$ is the jet energy and $p_{z}$ is the component of the jet momentum along the beam axis. As shown in [8], the production of dijets with invariant mass above $165 \mathrm{GeV}$ and $|y|$ less than 2.5 is also well described by NLO predictions. When

*e-mail: cms-publication-committee-chair@ cern.ch jets are well separated in rapidity, the description of the data becomes worse [2].

When the collision energy $\sqrt{s}$ is considerably larger than the hard scattering scale given by the jet transverse momentum, $p_{\mathrm{T}}$, the average number of produced jets grows rapidly, along with the phase space available in rapidity. This kinematic regime is expected to be described by the Balitsky-Fadin-Kuraev-Lipatov (BFKL) evolution [9-11] and $k_{\mathrm{T}}$ factorization [12-14]. An effective theory has been developed which describes strong interactions in this kinematic domain [15].

The ratio of the dijet production cross section in "inclusive" events to that in "exclusive" events, $R^{\text {incl }}=\sigma^{\text {incl }} / \sigma^{\text {excl }}$, as a function of the rapidity separation $|\Delta y|$ between two jets, is a sensitive probe of effects beyond collinear factorization [16]. Only jets with transverse momenta above a minimal value of $p_{\mathrm{T}}^{\mathrm{min}}$ are considered. Events with at least one pair of jets are denoted as "inclusive". Events with exactly one pair of jets are called "exclusive". In the inclusive case, the rapidity separation is evaluated for each pairwise combination of jets above threshold [16]. Mueller-Navelet jet pairs [17] are a subset of the inclusive dijet class. In this case only the jet at highest rapidity (i.e. most forward) and that at lowest rapidity (most backward) are considered. At low $|\Delta y|$, the inclusive dijet ratio, $R^{\text {incl }}$, is always larger than the corresponding Mueller-Navelet dijet ratio, $R^{\mathrm{MN}}=\sigma^{\mathrm{MN}} / \sigma^{\text {excl }}$, because all the jets in the rapidity interval between the Mueller-Navelet jets contribute to the inclusive cross section.

From the theoretical point of view, an advantage of the ratios $R^{\text {incl }}$ and $R^{\mathrm{MN}}$ with respect to the individual dijet production cross sections is that the influence of the uncertainty of the parton distribution functions is greatly reduced; in addition, the ratios are particularly sensitive to the parton radiation pattern [16]. At large enough energies, the parton subprocesses involve a large number of partons with compa- 
rable transverse energies. Such subprocesses, governed by BFKL evolution, lead to an increase of the ratios with increasing $|\Delta y|$ [16-18]. Experimentally, an additional advantage of the $R^{\text {incl }}$ and $R^{\mathrm{MN}}$ ratios defined above is the cancellation of most of the systematic uncertainties affecting both numerator and denominator.

Earlier measurements of Mueller-Navelet jets with $|\Delta y|$ up to 5 were made at the Tevatron by the D0 experiment $[19,20]$. D0 did not find indications of BFKL effects in the azimuthal decorrelation data [19]; however, a stronger than expected $\sqrt{s}$ dependence of the dijet production cross section was observed [20] when comparing the data at $\sqrt{s}=630$ and $1800 \mathrm{GeV}$ for dijets with large rapidity separation. The ATLAS Collaboration recently studied various dijet production cross section ratios at $\sqrt{s}=7 \mathrm{TeV}$ for $|\Delta y|<6$ [21]; the results are roughly in agreement with the DGLAP predictions. At the HERA ep collider, deviations from DGLAP were observed in studies of forward jets by the ZEUS [22, 23] and H1 [24, 25] Collaborations. However, no compelling evidence for BFKL effects was found.

The component of the CMS detector [26] most relevant for this analysis is the calorimeter system extending to pseudorapidities $|\eta|=5.2$, where $\eta=-\log [\tan (\theta / 2)]$, and $\theta$ is the polar angle relative to the anticlockwise proton beam direction. The crystal electromagnetic calorimeter (ECAL) and the brass/scintillator hadronic calorimeter (HCAL) extend to pseudorapidities $|\eta|=3.0$. The HCAL cells map to an array of ECAL crystals to form calorimeter towers projecting radially outwards from the nominal interaction point. The pseudorapidity region $3.0<|\eta|<5.2$ is covered by the hadronic forward (HF) calorimeter, which consists of steel absorber wedges with embedded radiation-hard quartz fibers, oriented parallel to the beam direction. The calorimeter towers in the barrel region have segmentation of $\Delta \eta \times \Delta \phi=0.087 \times 0.087$, becoming progressively larger in the endcap and forward regions $(\Delta \eta \times \Delta \phi=0.175 \times 0.175$ at $\eta \sim 4.5$ ).

The CMS trigger system consists of a hardware Level-1 trigger and a software high-level trigger. Jets formed online by the trigger system use ECAL, HCAL and HF inputs for energy clustering and are not corrected for the jet energy response. The minimum-bias trigger was defined as the coincidence of a signal from one of the two beam scintillation counters covering the range $3.23<|\eta|<4.65$ and a signal from one of the beam pick-up timing devices.

The data were collected in 2010, when LHC collided protons at $\sqrt{s}=7 \mathrm{TeV}$. Various triggers were used to cover the largest possible range in rapidity separation between jets. Dijets with moderate $|\Delta y|$ were selected by a single-jet trigger with a threshold on the raw (uncorrected) jet transverse momentum of $15 \mathrm{GeV}$. This trigger was significantly prescaled as the instantaneous luminosity increased, and the effective integrated luminosity recorded with it is $\simeq 33 \mathrm{nb}^{-1}$.
Dijet events with large rapidity separation are rare. Therefore, a dedicated trigger for forward-backward dijets was developed. This forward-backward-dijet trigger selects events with two jets in opposite hemispheres and $|\eta|>3.0$, and jet raw transverse momentum $p_{\mathrm{T}}>15 \mathrm{GeV}$. It was operated with moderate prescaling, and the effective integrated luminosity recorded with it is $\simeq 5 \mathrm{pb}^{-1}$. This allowed to collect a number of dijet events at high $|\Delta y|$ values more than 100 times larger than with the single-jet trigger.

The trigger efficiency was measured by means of a control sample selected with the minimum-bias trigger. The single-jet trigger was found to be $100 \%$ efficient for dijets with corrected $p_{\mathrm{T}}>35 \mathrm{GeV}$. The single-jet trigger was also used for the determination of the efficiency of the forwardbackward-dijet trigger. The latter was $100 \%$ efficient for dijets with $p_{\mathrm{T}}>35 \mathrm{GeV}$.

Jets were reconstructed offline from the energy depositions in the calorimeter towers, clustered with the anti- $k_{\mathrm{T}}$ algorithm [27, 28] with a distance parameter $R=0.5$. In the reconstruction process, the contribution from each tower was assigned a momentum, the absolute value and the direction of which were given by the energy measured in the tower, and the coordinates of the tower, respectively. The raw jet energy was obtained from the sum of the tower energies, and the raw jet momentum from the vectorial sum of the tower momenta. The raw jet energies were then corrected to establish a uniform relative response of the calorimeter in $\eta$ and a calibrated absolute response in transverse momentum $p_{\mathrm{T}}$ [29]. The jet energy resolution for calorimeter jets with $p_{\mathrm{T}} \sim 35 \mathrm{GeV}$ is about $22 \%$ for $|\eta|<0.5$ and about $10 \%$ for $4<|\eta|<4.5$ [30]. The uncertainty of the jet energy calibration for jets with $p_{\mathrm{T}} \sim 35 \mathrm{GeV}$ depends on $\eta$ and is $\simeq 7-8 \%$ [29].

In the offline analysis, at least one well-reconstructed primary vertex is required to be present within $\pm 24 \mathrm{~cm}$ of the nominal interaction point along the beamline [31]. In order to reduce the sensitivity to overlapping $p p$ collisions (the so-called "pile-up" events), events with only one primary vertex reconstructed within the luminous region were used for the measurement.

Loose jet quality cuts [32] were used to suppress the effect of calorimeter noise. Events with at least two jets with $p_{\mathrm{T}}>35 \mathrm{GeV}$ and $|y|<4.7$ were selected; only jets satisfying these criteria were used for the analysis. All pairwise combinations of jets from the selected events entered the inclusive distribution. For studies of Mueller-Navelet jets, only the pair consisting of the most forward and the most backward jet was considered. The exclusive dijet sample is a subset of the inclusive and Mueller-Navelet samples, and consists of events where exactly one pair of jets is found. The measured observables, $R^{\text {incl }}$ and $R^{\mathrm{MN}}$, are defined as the ratios of the yield of inclusive or Mueller-Navelet dijets to the yield of exclusive dijets in a specific $|\Delta y|$ bin, respectively. 
Detector effects were accounted for by applying bin-bybin corrections derived from Monte Carlo (MC) simulations. Simulated events produced with the generators PYTHIA6 (version 6.422) tune Z2 [33-35] and HERWIG++ [36] (version 2.4.2 with default settings) were passed through the full CMS detector simulation based on the GEANT4 package [37], and were input to the same event-reconstruction program as used for the data. To quantify detector effects, the distributions obtained from detector-level quantities were compared to the distributions obtained at the level of stable particles (lifetime $\tau$ such that $c \tau>10 \mathrm{~mm}$ ). The ratios of the stable-particle level and detector-level quantities in a given bin were used to correct the data.

Because of the finite $p_{\mathrm{T}}$ and $y$ resolutions, the detectorlevel distributions deviate from the corresponding ones at stable-particle level. Events can migrate to and from the exclusive or the inclusive samples because of fluctuations of the measured transverse jet momentum around the $p_{\mathrm{T}}^{\min }$ threshold. The amount of these migrations was estimated not to exceed $20 \%$. Similarly, the $|\Delta y|$ value measured at detector level may fall into a different $|\Delta y|$ bin compared to the stable-particle level; this effect, for the present data, is typically around 5-10\% and reaches $15-25 \%$ at most. The influence of these migrations on the measured ratios $R^{\text {incl }}$ and $R^{\mathrm{MN}}$ is minimal as the effects for numerator and denominator are similar. The uncorrected $R^{\mathrm{incl}}$ and $R^{\mathrm{MN}}$ ratios are reasonably well reproduced by the PYTHIA6 events and less well by HERWIG++ events, both of which have been passed through the detector simulation. The $p_{\mathrm{T}}$ and $\eta$ distributions of the jets at the detector level for both MC generators agree with the data within the jet energy scale uncertainty (see also [2]). The correction factors were therefore obtained with PYTHIA6, while their model dependence was estimated from the difference between the PYTHIA6 and HERWIG++ corrections.

The following sources of systematic effects were considered:

(1) Uncertainty of the jet energy calibration. The uncertainty of the measurement was estimated by shifting the jet energy scale (JES) by the $p_{T^{-}}$and $\eta$-dependent uncertainties derived in [29]. The resulting variation of the measurements does not exceed $4.2 \%$ for $R^{\text {incl }}$ and $3.8 \%$ for $R^{\mathrm{MN}}$.

(2) Uncertainty of the corrections for detector effects.

(a) Uncertainty due to model dependence of the correction factors. As discussed above, correction factors were determined by using HERWIG++ and PYTHIA6, and corrected measurements were obtained for each case. The difference in the results was taken as a measure of the model dependence of the correction factors; it does not exceed $3.4 \%$ for $R^{\text {incl }}$ and $3.3 \%$ for $R^{\mathrm{MN}}$.
Table 1 Sources of systematic effects and associated uncertainties. The ranges correspond to the variation of the uncertainty with $|\Delta y|$. For different uncertainty sources, the minimum and maximum values may correspond to different $|\Delta y|$ bins. For asymmetric uncertainties the upper and lower limits are shown

\begin{tabular}{lll}
\hline Source & $\begin{array}{l}R^{\text {incl }} \\
\text { uncertainty } \\
(\%)\end{array}$ & $\begin{array}{l}R^{\mathrm{MN}} \\
\text { uncertainty } \\
(\%)\end{array}$ \\
\hline Jet energy scale & $+(2.2-4.2)$ & $+(0.2-3.8)$ \\
& $-(1.0-3.0)$ & $-(0.2-2.3)$ \\
Uncertainty of detector corrections & $\pm(1.5-3.5)$ & $\pm(0.1-3.4)$ \\
Pile-up & $<1.3$ & $<1.3$ \\
Total & $+(2.8-5.6)$ & $+(0.2-4.8)$ \\
& $-(2.7-4.5)$ & $-(0.2-3.7)$ \\
\hline
\end{tabular}

(b) Uncertainty related to the quality of the MC description of the jet $p_{\mathrm{T}}$ and $y$ resolutions. These resolutions were modified by $\pm 10 \%$ in the simulation as recommended in [30]. A conservative estimate of the uncertainty associated to this effect does not exceed $\pm 1.0 \%$.

(3) An additional systematic effect may originate from the extra energy and jets due to pile-up collisions. As noted earlier, the measurement was restricted to events with only one reconstructed primary vertex to reduce the impact of pile-up. However, a contribution from interactions undetected because of vertex reconstruction inefficiency may still be present. By comparing data taken at different instantaneous luminosities, an upper limit of $1.3 \%$ was estimated for this effect. The value was obtained for pairs of jets in the forward region, where the impact of pile-up on jet reconstruction is more significant [29]; the effect is thus expected to be smaller for jets at central rapidities.

The total systematic uncertainty was calculated as the quadratic sum of the individual uncertainties. The total uncertainty is $|\Delta y|$ dependent but always smaller than $5.6 \%$ for $R^{\text {incl }}$ and $4.8 \%$ for $R^{\mathrm{MN}}$ (Table 1 ).

The measured ratios, corrected for detector effects, were compared to the predictions of several MC generators at the stable-particle level. A DGLAP leading-order parton shower approach is used in PYTHIA6 (version 6.422) tune Z2 [35], PYTHIA8 (version 8.145) [38] tune 4C [39] and HERWIG++ (version 2.5.1) tune UE-7000-EE-3 [36, 40]. For the simulation of the non-perturbative fragmentation, PYTHIA uses the Lund string model, and HERWIG++ the cluster fragmentation approach. The tunes mentioned above include multiple parton interactions (MPI) as a part of the underlying event (UE) modeling. The dijet observables might be affected by MPI through jets that do not originate from the same hard interaction. The effect was estimated by switching off the MPI options in PYTHIA6 and HERWIG++; no significant change in the predictions was observed. 

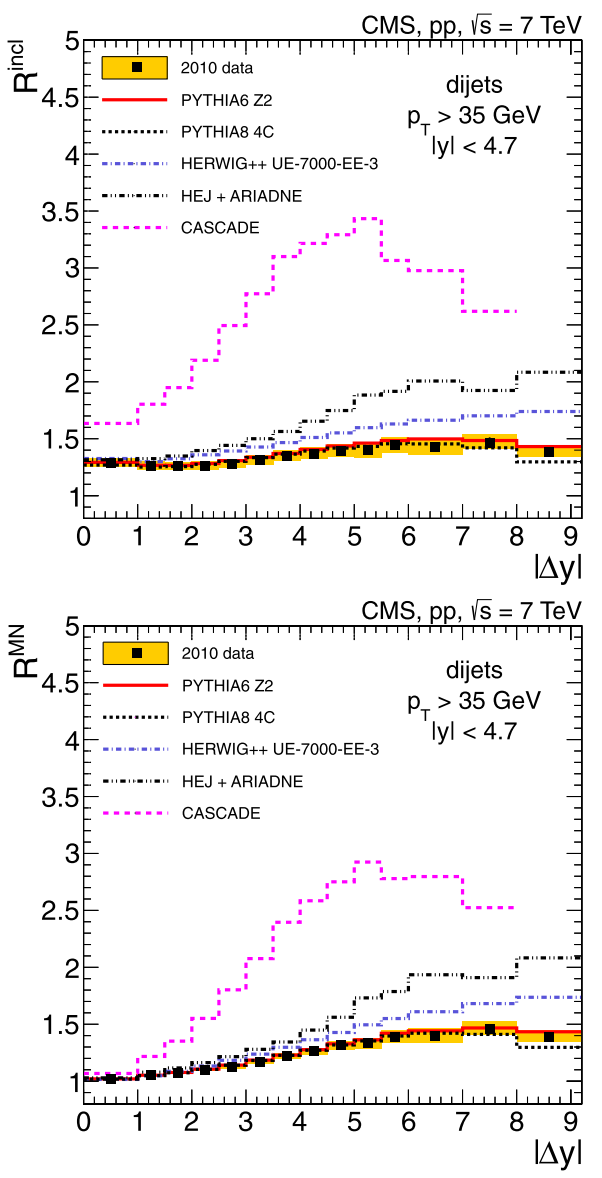

Fig. 1 Ratios of the inclusive to exclusive dijet cross sections as a function of the rapidity separation $|\Delta y|$ between the two jets, $R^{\text {incl }}$ (top panel) and $R^{\mathrm{MN}}$ (bottom panel), compared to the predictions of the DGLAP-based MC generators PYTHIA6, PYTHIA8 and HERWIG++, as well as of CASCADE and HEJ+ARIADNE, which incorporate elements of the BFKL approach. The shaded band indicates the size of the total systematic uncertainty of the data. Statistical uncertainties are smaller than the symbol sizes. Because of limitations in the CASCADE generator, it was not possible to obtain a reliable prediction for $|\Delta y|>8$

The Monte Carlo generators CASCADE (version 2.2.03) [41] and HEJ (version 1.3.2) [42] are motivated by the leading-logarithmic BFKL approach and incorporate parts of a next-to-leading logarithmic approximation. The HEJ generator produces parton-level jets; the corresponding showers were produced with the ARIADNE program [43]. The HEJ+ARIADNE package [44] version $0.99 \mathrm{~b}$, consisting of HEJ 1.3.2 and ARIADNE 4.12, was used.

The ratio $R^{\text {incl }}$ of inclusive to exclusive dijet production as a function of $|\Delta y|$ is presented in Fig. 1 (top panel). On average the inclusive cross section is $1.2-1.5$ times larger than the exclusive cross section. The ratio $R^{\text {incl }}$ grows with increasing $|\Delta y|$, as expected because of the larger phase space for hard parton radiation. At the highest $|\Delta y|, R^{\text {incl }}$ is expected to decrease because energy-momentum conservation suppresses the emission of extra jets. The $|\Delta y|$ value
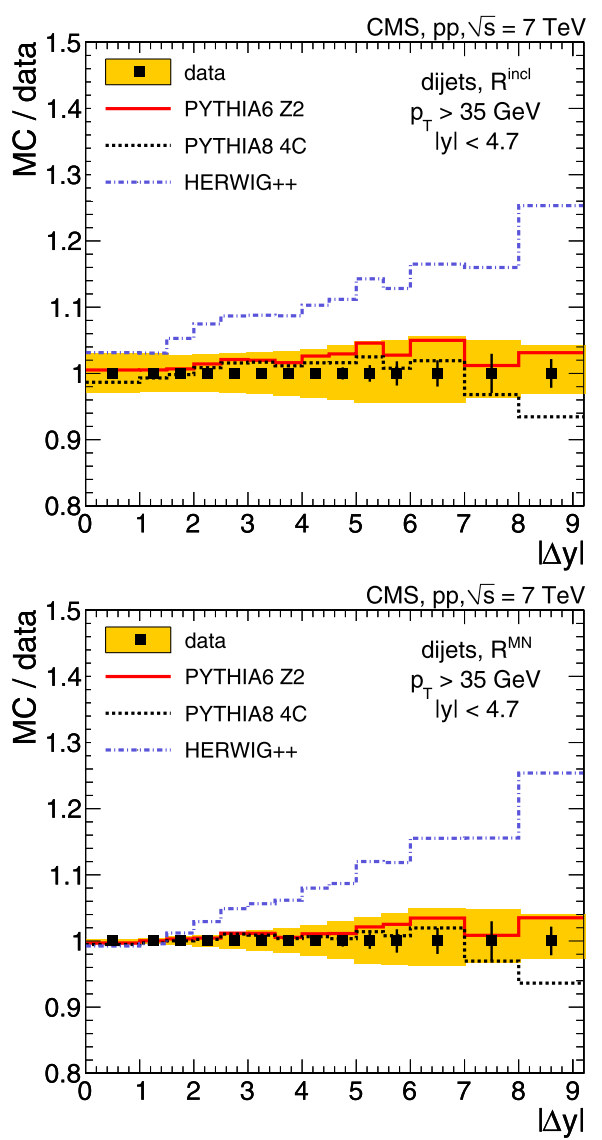

Fig. 2 Predictions for $R^{\text {incl }}$ (top) and $R^{\mathrm{MN}}$ (bottom) from DGLAPbased MC generators presented as ratio to data corrected for detector effects. Both BFKL-motivated generators CASCADE and HEJ+ARIADNE (not shown) lead to a MC/data ratio well above unity. The shaded band indicates the size of the total systematic uncertainty of the data while statistical uncertainties are shown as bars

where $R^{\text {incl }}$ starts to decrease varies from one MC generator to another, as can be seen in Fig. 1 .

The predictions from PYTHIA6 and PYTHIA8 agree with the measurement. HERWIG++ overestimates the ratio $R^{\text {incl }}$ at medium and large rapidity intervals. A detailed comparison between the data and the predictions of the DGLAPbased MC generators is presented as a ratio in Fig. 2 (top panel). It was checked explicitly that the results obtained from PYTHIA6 and HERWIG++ at parton level are close to the corresponding ones at stable-particle level. The different behaviour of PYTHIA6 and HERWIG++ is also observed at parton level.

The ratio $R^{\mathrm{MN}}$ and the corresponding MC to data ratio are presented in the bottom panels of Figs. 1-2. At large $|\Delta y|, R^{\mathrm{MN}}$ approaches $R^{\mathrm{incl}}$ as extra jet radiation contributing to $R^{\text {incl }}$ tends to concentrate at moderate rapidities. The quality of the predictions of the DGLAP-based MC generators for $R^{\mathrm{MN}}$ is similar to those for $R^{\text {incl }}$. The MC generators CASCADE and HEJ+ARIADNE considerably overestimate the measurements of both $R^{\text {incl }}$ and $R^{\mathrm{MN}}$. 
The ATLAS Collaboration measured several observables for dijets as functions of the rapidity separation between jets [21]. One is the fraction of events without jets with $p_{\mathrm{T}}$ above $p_{\mathrm{T}}^{\text {veto }}$ in the rapidity interval between the most forward and the most backward jets, which is equal to $1 / R^{\mathrm{MN}}$. A difference between data and the parton-level HEJ prediction at large $|\Delta y|$ for $70<\left\langle p_{\mathrm{T}}\right\rangle<90 \mathrm{GeV}, p_{\mathrm{T}}^{\text {veto }}=\left\langle p_{\mathrm{T}}\right\rangle$ and $|\Delta y|<6$ was reported (Fig. 8 from [21]), which is in qualitative agreement with the result presented here.

To conclude, the first measurement of the ratios $R^{\text {incl }}$ and $R^{\mathrm{MN}}$ in a wide range of rapidity separation, up to $|\Delta y|=9.2$, in proton-proton collisions at $\sqrt{s}=7 \mathrm{TeV}$ was presented. A moderate rise of the ratio of the inclusive to exclusive dijet production cross sections as a function of $|\Delta y|$ is observed. The predictions of the PYTHIA6 and PYTHIA8 generators agree with the measurements. The predictions of the HERWIG++ generator are larger than the measurement especially at large $|\Delta y|$. The BFKL-motivated generators CASCADE and HEJ+ARIADNE predict for these ratios a significantly stronger rise than observed. The moderate rise of the measured dijet ratios indicates that the BFKL effects are not dominant for jets with $p_{\mathrm{T}}>35 \mathrm{GeV}$ at the present collision energy of $7 \mathrm{TeV}$.

\begin{abstract}
Acknowledgements We congratulate our colleagues in the CERN accelerator departments for the excellent performance of the LHC machine. We thank the technical and administrative staff at CERN and other CMS institutes, and acknowledge support from: FMSR (Austria); FNRS and FWO (Belgium); CNPq, CAPES, FAPERJ, and FAPESP (Brazil); MES (Bulgaria); CERN; CAS, MoST, and NSFC (China); COLCIENCIAS (Colombia); MSES (Croatia); RPF (Cyprus); MoER, SF0690030s09 and ERDF (Estonia); Academy of Finland, MEC, and HIP (Finland); CEA and CNRS/IN2P3 (France); BMBF, DFG, and HGF (Germany); GSRT (Greece); OTKA and NKTH (Hungary); DAE and DST (India); IPM (Iran); SFI (Ireland); INFN (Italy); NRF and WCU (Korea); LAS (Lithuania); CINVESTAV, CONACYT, SEP, and UASLP-FAI (Mexico); MSI (New Zealand); PAEC (Pakistan); MSHE and NSC (Poland); FCT (Portugal); JINR (Armenia, Belarus, Georgia, Ukraine, Uzbekistan); MON, RosAtom, RAS and RFBR (Russia); MSTD (Serbia); MICINN and CPAN (Spain); Swiss Funding Agencies (Switzerland); NSC (Taipei); TUBITAK and TAEK (Turkey); STFC (United Kingdom); DOE and NSF (USA).
\end{abstract}

Open Access This article is distributed under the terms of the Creative Commons Attribution License which permits any use, distribution, and reproduction in any medium, provided the original author(s) and the source are credited.

\section{References}

1. CMS Collaboration, Measurement of the inclusive jet cross section in pp collisions at $\sqrt{s}=7 \mathrm{TeV}$. Phys. Rev. Lett. 107, 132001 (2011). doi:10.1103/PhysRevLett.107.132001, arXiv:1106.0208

2. CMS Collaboration, Measurement of the inclusive production cross sections for forward jets and for dijet events with one for- ward and one central jet in pp collisions at $\sqrt{s}=7 \mathrm{TeV}$. J. High Energy Phys. 1206, 036 (2012). doi:10.1007/JHEP06(2012)036, arXiv: 1202.0704

3. V.N. Gribov, L.N. Lipatov, Deep inelastic ep scattering in perturbation theory. Sov. J. Nucl. Phys. 15, 438 (1972)

4. V.N. Gribov, L.N. Lipatov, $e^{+} e^{-}$pair annihilation and deep inelastic ep scattering in perturbation theory. Sov. J. Nucl. Phys. 15, 675 (1972)

5. L.N. Lipatov, The parton model and perturbation theory. Sov. J. Nucl. Phys. 20, 94 (1975)

6. G. Altarelli, G. Parisi, Asymptotic freedom in parton language. Nucl. Phys. B 126, 298 (1977). doi:10.1016/05503213(77)90384-4

7. Y.L. Dokshitzer, Calculation of the structure functions for deep inelastic scattering and $e^{+} e^{-}$annihilation by perturbation theory in quantum chromodynamics. Sov. Phys. JETP 46, 641 (1977)

8. CMS Collaboration, Measurement of the differential dijet production cross section in proton-proton collisions at $\sqrt{s}=7 \mathrm{TeV}$. Phys. Lett. B 700, 187 (2011). doi:10.1016/j.physletb.2011.05. 027, arXiv: 1104.1693

9. E.A. Kuraev, L.N. Lipatov, V.S. Fadin, Multi-reggeon processes in the Yang-Mills theory. Sov. Phys. JETP 44, 443 (1976)

10. E.A. Kuraev, L.N. Lipatov, V.S. Fadin, The Pomeranchuk singularity in nonabelian gauge theories. Sov. Phys. JETP 45, 199 (1977)

11. I.I. Balitsky, L.N. Lipatov, The Pomeranchuk singularity in quantum chromodynamics. Sov. J. Nucl. Phys. 28, 822 (1978)

12. E.M. Levin et al., Heavy quark production in semihard nucleon interactions. Sov. J. Nucl. Phys. 53, 657 (1991)

13. S. Catani, M. Ciafaloni, F. Hautmann, High-energy factorization and small-x heavy flavour production. Nucl. Phys. B 366, 135 (1991). doi:10.1016/0550-3213(91)90055-3

14. J.C. Collins, R.K. Ellis, Heavy quark production in very highenergy hadron collisions. Nucl. Phys. B 360, 3 (1991). doi:10. 1016/0550-3213(91)90288-9

15. L. Lipatov, Small $x$ physics in perturbative QCD. Phys. Rep. 286, 131-198 (1997). doi:10.1016/S0370-1573(96)00045-2, arXiv: hep-ph/9610276

16. V.T. Kim, G.B. Pivovarov, BFKL QCD pomeron in high-energy hadron collisions: inclusive dijet production. Phys. Rev. D 53, 6 (1996). doi:10.1103/PhysRevD.53.R6, arXiv:hep-ph/9506381

17. A.H. Mueller, H. Navelet, An inclusive minijet cross-section and the bare pomeron in QCD. Nucl. Phys. B 282, 727 (1987). doi:10. 1016/0550-3213(87)90705-X

18. B. Andersson et al., Small x phenomenology: summary and status. Eur. Phys. J. C 25, 77 (2002). doi:10.1007/s10052-002-0998-7, arXiv:hep-ph/0204115

19. D0 Collaboration, The azimuthal decorrelation of jets widely separated in rapidity. Phys. Rev. Lett. 77, 595 (1996). doi:10.1103/ PhysRevLett.77.595, arXiv:hep-ex/9603010

20. D0 Collaboration, Probing BFKL dynamics in the dijet cross section at large rapidity intervals in $p \bar{p}$ collisions at $\sqrt{s}=$ $1800 \mathrm{GeV}$ and 630-GeV. Phys. Rev. Lett. 84, 5722 (2000). doi:10. 1103/PhysRevLett.84.5722, arXiv:hep-ex/9912032

21. ATLAS Collaboration, Measurement of dijet production with a veto on additional central jet activity in $p p$ collisions with $\sqrt{s}=$ $7 \mathrm{TeV}$ using the ATLAS detector. J. High Energy Phys. 09, 053 (2011). doi:10.1007/JHEP09(2011)053, arXiv:1107.1641v2.

22. ZEUS Collaboration, Forward jet production in deep inelastic scattering at HERA. Eur. Phys. J. C 6, 239 (1999). doi:10.1007/ s100529801018, arXiv:hep-ex/9805016v1

23. ZEUS Collaboration, Forward-jet production in deep inelastic ep scattering at HERA. Eur. Phys. J. C 52, 515 (2007). doi:10.1140/ epjc/s10052-007-0418-0, arXiv:0707.3093 
24. H1 Collaboration, Transverse energy and forward jet production in the low x regime at HERA. Phys. Lett. B 356, 118 (1995). doi:10.1016/0370-2693(95)00804-T

25. H1 Collaboration, Measurement of the azimuthal correlation between the most forward jet and the scattered positron in deepinelastic scattering at HERA. Eur. Phys. J. C 52, 1910 (2012). doi:10.1140/epjc/s10052-012-1910-8, arXiv:1111.4227

26. CMS Collaboration, The CMS experiment at the CERN LHC. J. Instrum. 03, S08004 (2008). doi:10.1088/1748-0221/3/08/ S08004

27. M. Cacciari, G.P. Salam, G. Soyez, The anti-kt jet clustering algorithm. J. High Energy Phys. 04, 063 (2008). doi:10.1088/11266708/2008/04/063, arXiv:0802.1189

28. M. Cacciari, G.P. Salam, Dispelling the $N^{3}$ myth for the kT jetfinder. Phys. Lett. B 641, 57 (2006). doi:10.1016/j.physletb.2006. 08.037, arXiv:hep-ph/0512210

29. CMS Collaboration, Determination of jet energy calibration and transverse momentum resolution in CMS. J. Instrum. 6, P11002 (2011). doi:10.1088/1748-0221/6/11/P11002, arXiv:1107.4277

30. CMS Collaboration, Jet performance in $p p$ collisions at $\sqrt{s}=$ $7 \mathrm{TeV}$, CMS physics analysis summary CMS-PAS-JME-10-003 (2010)

31. CMS Collaboration, Tracking and primary vertex results in first $7 \mathrm{TeV}$ collisions, CMS physics analysis summary CMS-PASTRK-10-005 (2010)

32. CMS Collaboration, Calorimeter jet quality criteria for the first CMS collision data, CMS physics analysis summary CMS-PASJME-09-008 (2010)

33. T. Sjöstrand, S. Mrenna, P. Skands, Pythia 6.4 physics and manual. J. High Energy Phys. 05, 026 (2006). doi:10.1088/1126-6708/ 2006/05/026
34. R. Field, Early LHC underlying event data—findings and surprises (2010). arXiv:1010.3558

35. CMS Collaboration, Measurement of the underlying event activity at the LHC with $\sqrt{s}=7 \mathrm{TeV}$ and comparison with $\sqrt{s}=$ 0.9 TeV. J. High Energy Phys. 09, 109 (2011). doi:10.1007/ JHEP09(2011)109, arXiv:1107.0330

36. M. Bähr et al., HERWIG++ physics and manual. Eur. Phys. J. C 58, 639 (2008). doi:10.1140/epjc/s10052-008-0798-9

37. S. Agostinelli et al., Geant4-a simulation toolkit. Nucl. Instrum. Methods A 506, 250 (2003). doi:10.1016/S01689002(03)01368-8

38. T. Sjöstrand, S. Mrenna, P. Skands, A brief introduction to PYTHIA 8.1. Comput. Phys. Commun. 178, 852 (2008). doi:10. 1016/j.cpc.2008.01.036

39. R. Corke, T. Sjöstrand, Interleaved parton showers and tuning prospects. J. High Energy Phys. 3, 32 (2011). doi:10.1007/ JHEP03(2011)032, arXiv:1011.1759

40. S. Gieseke et al., HERWIG++ 2.5 release note, arXiv:1102. $1672 \mathrm{v} 1$

41. H. Jung et al., The CCFM Monte Carlo generator CASCADE version 2.2.03. Eur. Phys. J. C 70, 1237 (2010). doi:10.1140/epjc/ s10052-010-1507-z, arXiv:1008.0152

42. J.R. Andersen, J.M. Smillie, Multiple jets at the LHC with high energy jets. J. High Energy Phys. 06, 010 (2011). doi:10.1007/ JHEP06(2011)010, arXiv:1101.5394

43. L. Lönnblad, Ariadne version 4: a program for simulation of QCD cascades implementing the colour dipole model. Comput. Phys. Commun. 71, 15 (1992). doi:10.1016/0010-4655(92)90068-A

44. J. Andersen, L. Lonnblad, J. Smillie, A parton shower for high energy jets. J. High Energy Phys. 7, 110 (2011). doi:10.1007/ JHEP07(2011)110, arXiv:1104.1316

\section{The CMS Collaboration}

\section{Yerevan Physics Institute, Yerevan, Armenia}

S. Chatrchyan, V. Khachatryan, A.M. Sirunyan, A. Tumasyan

\section{Institut für Hochenergiephysik der OeAW, Wien, Austria}

W. Adam, T. Bergauer, M. Dragicevic, J. Erö, C. Fabjan, M. Friedl, R. Frühwirth, V.M. Ghete, J. Hammer ${ }^{1}$, M. Hoch, N. Hörmann, J. Hrubec, M. Jeitler, W. Kiesenhofer, M. Krammer, D. Liko, I. Mikulec, M. Pernicka ${ }^{\dagger}$, B. Rahbaran, C. Rohringer, H. Rohringer, R. Schöfbeck, J. Strauss, A. Taurok, F. Teischinger, P. Wagner, W. Waltenberger, G. Walzel, E. Widl, C.-E. Wulz

\section{National Centre for Particle and High Energy Physics, Minsk, Belarus}

V. Mossolov, N. Shumeiko, J. Suarez Gonzalez

\section{Universiteit Antwerpen, Antwerpen, Belgium}

S. Bansal, L. Benucci, T. Cornelis, E.A. De Wolf, X. Janssen, S. Luyckx, T. Maes, L. Mucibello, S. Ochesanu, B. Roland, R. Rougny, M. Selvaggi, H. Van Haevermaet, P. Van Mechelen, N. Van Remortel, A. Van Spilbeeck

\section{Vrije Universiteit Brussel, Brussel, Belgium}

F. Blekman, S. Blyweert, J. D’Hondt, R. Gonzalez Suarez, A. Kalogeropoulos, M. Maes, A. Olbrechts, W. Van Doninck, P. Van Mulders, G.P. Van Onsem, I. Villella

\section{Université Libre de Bruxelles, Bruxelles, Belgium}

O. Charaf, B. Clerbaux, G. De Lentdecker, V. Dero, A.P.R. Gay, G.H. Hammad, T. Hreus, A. Léonard, P.E. Marage, L. Thomas, C. Vander Velde, P. Vanlaer, J. Wickens 


\section{Ghent University, Ghent, Belgium}

V. Adler, K. Beernaert, A. Cimmino, S. Costantini, G. Garcia, M. Grunewald, B. Klein, J. Lellouch, A. Marinov, J. Mccartin, A.A. Ocampo Rios, D. Ryckbosch, N. Strobbe, F. Thyssen, M. Tytgat, L. Vanelderen, P. Verwilligen, S. Walsh, E. Yazgan, N. Zaganidis

Université Catholique de Louvain, Louvain-la-Neuve, Belgium

S. Basegmez, G. Bruno, L. Ceard, J. De Favereau De Jeneret, C. Delaere, T. du Pree, D. Favart, L. Forthomme, A. Giammanco ${ }^{2}$, G. Grégoire, J. Hollar, V. Lemaitre, J. Liao, O. Militaru, C. Nuttens, D. Pagano, A. Pin, K. Piotrzkowski, N. Schul

Université de Mons, Mons, Belgium

N. Beliy, T. Caebergs, E. Daubie

Centro Brasileiro de Pesquisas Fisicas, Rio de Janeiro, Brazil

G.A. Alves, M. Correa Martins Junior, D. De Jesus Damiao, T. Martins, M.E. Pol, M.H.G. Souza

Universidade do Estado do Rio de Janeiro, Rio de Janeiro, Brazil

W.L. Aldá Júnior, W. Carvalho, A. Custódio, E.M. Da Costa, C. De Oliveira Martins, S. Fonseca De Souza, D. Matos Figueiredo, L. Mundim, H. Nogima, V. Oguri, W.L. Prado Da Silva, A. Santoro, S.M. Silva Do Amaral, L. Soares Jorge, A. Sznajder

Instituto de Fisica Teorica, Universidade Estadual Paulista, Sao Paulo, Brazil

T.S. Anjos $^{3}$, C.A. Bernardes ${ }^{3}$, F.A. Dias ${ }^{4}$, T.R. Fernandez Perez Tomei, E.M. Gregores ${ }^{3}$, C. Lagana, F. Marinho, P.G. Mercadante ${ }^{3}$, S.F. Novaes, S.S. Padula

Institute for Nuclear Research and Nuclear Energy, Sofia, Bulgaria

V. Genchev ${ }^{1}$, P. Iaydjiev ${ }^{1}$, S. Piperov, M. Rodozov, S. Stoykova, G. Sultanov, V. Tcholakov, R. Trayanov, M. Vutova

University of Sofia, Sofia, Bulgaria

A. Dimitrov, R. Hadjiiska, A. Karadzhinova, V. Kozhuharov, L. Litov, B. Pavlov, P. Petkov

Institute of High Energy Physics, Beijing, China

J.G. Bian, G.M. Chen, H.S. Chen, C.H. Jiang, D. Liang, S. Liang, X. Meng, J. Tao, J. Wang, J. Wang, X. Wang, Z. Wang, H. Xiao, M. Xu, J. Zang, Z. Zhang

State Key Lab. of Nucl. Phys. and Tech., Peking University, Beijing, China

C. Asawatangtrakuldee, Y. Ban, S. Guo, Y. Guo, W. Li, S. Liu, Y. Mao, S.J. Qian, H. Teng, S. Wang, B. Zhu, W. Zou

Universidad de Los Andes, Bogota, Colombia

A. Cabrera, B. Gomez Moreno, A.F. Osorio Oliveros, J.C. Sanabria

Technical University of Split, Split, Croatia

N. Godinovic, D. Lelas, R. Plestina ${ }^{5}$, D. Polic, I. Puljak ${ }^{1}$

University of Split, Split, Croatia

Z. Antunovic, M. Dzelalija, M. Kovac

Institute Rudjer Boskovic, Zagreb, Croatia

V. Brigljevic, S. Duric, K. Kadija, J. Luetic, S. Morovic

University of Cyprus, Nicosia, Cyprus

A. Attikis, M. Galanti, J. Mousa, C. Nicolaou, F. Ptochos, P.A. Razis

Charles University, Prague, Czech Republic

M. Finger, M. Finger Jr.

Academy of Scientific Research and Technology of the Arab Republic of Egypt, Egyptian Network of High Energy Physics, Cairo, Egypt

Y. Assran ${ }^{6}$, A. Ellithi Kamel ${ }^{7}$, S. Khalil ${ }^{8}$, M.A. Mahmoud ${ }^{9}$, A. Radi ${ }^{8,10}$

National Institute of Chemical Physics and Biophysics, Tallinn, Estonia

A. Hektor, M. Kadastik, M. Müntel, M. Raidal, L. Rebane, A. Tiko 
Department of Physics, University of Helsinki, Helsinki, Finland

V. Azzolini, P. Eerola, G. Fedi, M. Voutilainen

Helsinki Institute of Physics, Helsinki, Finland

S. Czellar, J. Härkönen, A. Heikkinen, V. Karimäki, R. Kinnunen, M.J. Kortelainen, T. Lampén, K. Lassila-Perini, S. Lehti, T. Lindén, P. Luukka, T. Mäenpää, T. Peltola, E. Tuominen, J. Tuominiemi, E. Tuovinen, D. Ungaro, L. Wendland

Lappeenranta University of Technology, Lappeenranta, Finland

K. Banzuzi, A. Korpela, T. Tuuva

Laboratoire d'Annecy-le-Vieux de Physique des Particules, IN2P3-CNRS, Annecy-le-Vieux, France

D. Sillou

\section{DSM/IRFU, CEA/Saclay, Gif-sur-Yvette, France}

M. Besancon, S. Choudhury, M. Dejardin, D. Denegri, B. Fabbro, J.L. Faure, F. Ferri, S. Ganjour, A. Givernaud, P. Gras, G. Hamel de Monchenault, P. Jarry, E. Locci, J. Malcles, L. Millischer, J. Rander, A. Rosowsky, I. Shreyber, M. Titov

Laboratoire Leprince-Ringuet, Ecole Polytechnique, IN2P3-CNRS, Palaiseau, France

S. Baffioni, F. Beaudette, L. Benhabib, L. Bianchini, M. Bluj ${ }^{11}$, C. Broutin, P. Busson, C. Charlot, N. Daci, T. Dahms, L. Dobrzynski, S. Elgammal, R. Granier de Cassagnac, M. Haguenauer, P. Miné, C. Mironov, C. Ochando, P. Paganini, D. Sabes, R. Salerno, Y. Sirois, C. Thiebaux, C. Veelken, A. Zabi

Institut Pluridisciplinaire Hubert Curien, Université de Strasbourg, Université de Haute Alsace Mulhouse, CNRS/IN2P3, Strasbourg, France

J.-L. Agram ${ }^{12}$, J. Andrea, D. Bloch, D. Bodin, J.-M. Brom, M. Cardaci, E.C. Chabert, C. Collard, E. Conte ${ }^{12}$, F. Drouhin ${ }^{12}$, C. Ferro, J.-C. Fontaine ${ }^{12}$, D. Gelé, U. Goerlach, P. Juillot, M. Karim ${ }^{12}$, A.-C. Le Bihan, P. Van Hove

Centre de Calcul de l'Institut National de Physique Nucleaire et de Physique des Particules (IN2P3), Villeurbanne, France

F. Fassi, D. Mercier

Université de Lyon, Université Claude Bernard Lyon 1, CNRS-IN2P3, Institut de Physique Nucléaire de Lyon, Villeurbanne, France

C. Baty, S. Beauceron, N. Beaupere, M. Bedjidian, O. Bondu, G. Boudoul, D. Boumediene, H. Brun, J. Chasserat, R. Chierici ${ }^{1}$, D. Contardo, P. Depasse, H. El Mamouni, A. Falkiewicz, J. Fay, S. Gascon, M. Gouzevitch, B. Ille, T. Kurca, T. Le Grand, M. Lethuillier, L. Mirabito, S. Perries, V. Sordini, S. Tosi, Y. Tschudi, P. Verdier, S. Viret

Institute of High Energy Physics and Informatization, Tbilisi State University, Tbilisi, Georgia

D. Lomidze

RWTH Aachen University, I. Physikalisches Institut, Aachen, Germany

G. Anagnostou, S. Beranek, M. Edelhoff, L. Feld, N. Heracleous, O. Hindrichs, R. Jussen, K. Klein, J. Merz, A. Ostapchuk, A. Perieanu, F. Raupach, J. Sammet, S. Schael, D. Sprenger, H. Weber, B. Wittmer, V. Zhukov ${ }^{13}$

RWTH Aachen University, III. Physikalisches Institut A, Aachen, Germany

M. Ata, J. Caudron, E. Dietz-Laursonn, M. Erdmann, A. Güth, T. Hebbeker, C. Heidemann, K. Hoepfner, T. Klimkovich, D. Klingebiel, P. Kreuzer, D. Lanske ${ }^{\dagger}$, J. Lingemann, C. Magass, M. Merschmeyer, A. Meyer, M. Olschewski, P. Papacz, H. Pieta, H. Reithler, S.A. Schmitz, L. Sonnenschein, J. Steggemann, D. Teyssier, M. Weber

RWTH Aachen University, III. Physikalisches Institut B, Aachen, Germany

M. Bontenackels, V. Cherepanov, M. Davids, G. Flügge, H. Geenen, M. Geisler, W. Haj Ahmad, F. Hoehle, B. Kargoll, T. Kress, Y. Kuessel, A. Linn, A. Nowack, L. Perchalla, O. Pooth, J. Rennefeld, P. Sauerland, A. Stahl, M.H. Zoeller

Deutsches Elektronen-Synchrotron, Hamburg, Germany

M. Aldaya Martin, W. Behrenhoff, U. Behrens, M. Bergholz ${ }^{14}$, A. Bethani, K. Borras, A. Burgmeier, A. Cakir, L. Calligaris, A. Campbell, E. Castro, D. Dammann, G. Eckerlin, D. Eckstein, A. Flossdorf, G. Flucke, A. Geiser, J. Hauk, H. Jung ${ }^{1}$, M. Kasemann, P. Katsas, C. Kleinwort, H. Kluge, A. Knutsson, M. Krämer, D. Krücker, E. Kuznetsova, W. Lange, W. Lohmann ${ }^{14}$, B. Lutz, R. Mankel, I. Marfin, M. Marienfeld, I.-A. Melzer-Pellmann, A.B. Meyer, J. Mnich, A. Mussgiller, S. Naumann-Emme, J. Olzem, A. Petrukhin, D. Pitzl, A. Raspereza, P.M. Ribeiro Cipriano, M. Rosin, J. Salfeld-Nebgen, R. Schmidt ${ }^{14}$, T. Schoerner-Sadenius, N. Sen, A. Spiridonov, M. Stein, J. Tomaszewska, R. Walsh, C. Wissing 
University of Hamburg, Hamburg, Germany

C. Autermann, V. Blobel, S. Bobrovskyi, J. Draeger, H. Enderle, J. Erfle, U. Gebbert, M. Görner, T. Hermanns, R.S. Höing, K. Kaschube, G. Kaussen, H. Kirschenmann, R. Klanner, J. Lange, B. Mura, F. Nowak, N. Pietsch, C. Sander, H. Schettler, P. Schleper, E. Schlieckau, A. Schmidt, M. Schröder, T. Schum, H. Stadie, G. Steinbrück, J. Thomsen

Institut für Experimentelle Kernphysik, Karlsruhe, Germany

C. Barth, J. Berger, T. Chwalek, W. De Boer, A. Dierlamm, G. Dirkes, M. Feindt, J. Gruschke, M. Guthoff ${ }^{1}$, C. Hackstein, F. Hartmann, M. Heinrich, H. Held, K.H. Hoffmann, S. Honc, I. Katkov ${ }^{13}$, J.R. Komaragiri, T. Kuhr, D. Martschei, S. Mueller, Th. Müller, M. Niegel, A. Nürnberg, O. Oberst, A. Oehler, J. Ott, T. Peiffer, G. Quast, K. Rabbertz, F. Ratnikov, N. Ratnikova, M. Renz, S. Röcker, C. Saout, A. Scheurer, P. Schieferdecker, F.-P. Schilling, M. Schmanau, G. Schott, H.J. Simonis, F.M. Stober, D. Troendle, J. Wagner-Kuhr, T. Weiler, M. Zeise, E.B. Ziebarth

Institute of Nuclear Physics "Demokritos", Aghia Paraskevi, Greece

G. Daskalakis, T. Geralis, S. Kesisoglou, A. Kyriakis, D. Loukas, I. Manolakos, A. Markou, C. Markou, C. Mavrommatis, E. Ntomari

University of Athens, Athens, Greece

L. Gouskos, T.J. Mertzimekis, A. Panagiotou, N. Saoulidou, E. Stiliaris

University of Ioánnina, Ioánnina, Greece

I. Evangelou, C. Foudas ${ }^{1}$, P. Kokkas, N. Manthos, I. Papadopoulos, V. Patras, F.A. Triantis

KFKI Research Institute for Particle and Nuclear Physics, Budapest, Hungary

A. Aranyi, G. Bencze, L. Boldizsar, C. Hajdu ${ }^{1}$, P. Hidas, D. Horvath ${ }^{15}$, A. Kapusi, K. Krajczar ${ }^{16}$, F. Sikler ${ }^{1}$, V. Veszpremi, G. Vesztergombi ${ }^{16}$

Institute of Nuclear Research ATOMKI, Debrecen, Hungary

N. Beni, J. Molnar, J. Palinkas, Z. Szillasi

University of Debrecen, Debrecen, Hungary

J. Karancsi, P. Raics, Z.L. Trocsanyi, B. Ujvari

Panjab University, Chandigarh, India

S.B. Beri, V. Bhatnagar, N. Dhingra, R. Gupta, M. Jindal, M. Kaur, J.M. Kohli, M.Z. Mehta, N. Nishu, L.K. Saini, A. Sharma, A.P. Singh, J. Singh, S.P. Singh

University of Delhi, Delhi, India

S. Ahuja, B.C. Choudhary, A. Kumar, A. Kumar, S. Malhotra, M. Naimuddin, K. Ranjan, V. Sharma, R.K. Shivpuri

Saha Institute of Nuclear Physics, Kolkata, India

S. Banerjee, S. Bhattacharya, S. Dutta, B. Gomber, Sa. Jain, Sh. Jain, R. Khurana, S. Sarkar

Bhabha Atomic Research Centre, Mumbai, India

R.K. Choudhury, D. Dutta, S. Kailas, V. Kumar, A.K. Mohanty ${ }^{1}$, L.M. Pant, P. Shukla

Tata Institute of Fundamental Research - EHEP, Mumbai, India

T. Aziz, S. Ganguly, M. Guchait ${ }^{17}$, A. Gurtu ${ }^{18}$, M. Maity ${ }^{19}$, G. Majumder, K. Mazumdar, G.B. Mohanty, B. Parida, A. Saha, K. Sudhakar, N. Wickramage

Tata Institute of Fundamental Research - HECR, Mumbai, India

S. Banerjee, S. Dugad, N.K. Mondal

Institute for Research in Fundamental Sciences (IPM), Tehran, Iran

H. Arfaei, H. Bakhshiansohi ${ }^{20}$, S.M. Etesami ${ }^{21}$, A. Fahim ${ }^{20}$, M. Hashemi, H. Hesari, A. Jafari ${ }^{20}$, M. Khakzad, A. Mohammadi ${ }^{22}$, M. Mohammadi Najafabadi, S. Paktinat Mehdiabadi, B. Safarzadeh ${ }^{23}$, M. Zeinali ${ }^{21}$

INFN Sezione di Bari ${ }^{\mathrm{a}}$, Università di Bari ${ }^{\mathrm{b}}$, Politecnico di Bari ${ }^{\mathrm{c}}$, Bari, Italy

M. Abbrescia ${ }^{a, b}$, L. Barbone ${ }^{a, b}$, C. Calabria ${ }^{a, b}$, S.S. Chhibra ${ }^{a, b}$, A. Colaleo ${ }^{a}$, D. Creanza ${ }^{a, c}$, N. De Filippis ${ }^{a, c, 1}$, M. De Palma ${ }^{a, b}$, L. Fiore ${ }^{a}$, G. Iaselli ${ }^{a, c}$, L. Lusito ${ }^{a, b}$, G. Maggi ${ }^{a, c}$, M. Maggi ${ }^{a}$, N. Manna ${ }^{a, b}$, B. Marangellia ${ }^{a, b}$, S. My ${ }^{a, c}$, S. Nuzzo ${ }^{a, b}$, N. Pacifico ${ }^{a, b}$, A. Pompili ${ }^{a, b}$, G. Pugliese ${ }^{a, c}$, F. Romano ${ }^{a, c}$, G. Selvaggi ${ }^{a, b}$, L. Silvestris ${ }^{a}$, G. Singh ${ }^{a, b}$, S. Tupputi ${ }^{\mathrm{a}, \mathrm{b}}$, G. Zito ${ }^{\mathrm{a}}$ 
INFN Sezione di Bologna ${ }^{a}$, Università di Bologna ${ }^{b}$, Bologna, Italy

G. Abbiendi ${ }^{\mathrm{a}}$, A.C. Benvenuti ${ }^{\mathrm{a}}$, D. Bonacorsi ${ }^{\mathrm{a}}$, S. Braibant-Giacomellia ${ }^{\mathrm{a}, \mathrm{b}}$, L. Brigliadori $^{\mathrm{a}}$, P. Capiluppi ${ }^{\mathrm{a}, \mathrm{b}}$, A. Castro $^{\mathrm{a}, \mathrm{b}}$, F.R. Cavallo ${ }^{\mathrm{a}}$, M. Cuffiani ${ }^{\mathrm{a}, \mathrm{b}}$, G.M. Dallavalle ${ }^{\mathrm{a}}$, F. Fabbri ${ }^{\mathrm{a}}$, A. Fanfani ${ }^{\mathrm{a}, \mathrm{b}}$, D. Fasanella ${ }^{\mathrm{a}, 1}$, P. Giacomelli ${ }^{\mathrm{a}}$, C. Grandia, S. Marcellini ${ }^{\mathrm{a}}$, G. Masetti ${ }^{\mathrm{a}}$, M. Meneghelli ${ }^{\mathrm{a}} \mathrm{b}$, A. Montanaria ${ }^{\mathrm{a}}$, F.L. Navarria ${ }^{\mathrm{a}, \mathrm{b}}$, F. Odorici ${ }^{\mathrm{a}}$, A. Perrotta ${ }^{\mathrm{a}}$, F. Primavera ${ }^{\mathrm{a}}$, A.M. Rossi ${ }^{\mathrm{a}, \mathrm{b}}, \mathrm{T}$. Rovelli ${ }^{\mathrm{a}, \mathrm{b}}$, G. Siroli ${ }^{\mathrm{a}, \mathrm{b}}$, R. Travaglini $\mathrm{i}^{\mathrm{a}, \mathrm{b}}$

INFN Sezione di Catania ${ }^{\mathrm{a}}$, Università di Catania ${ }^{\mathrm{b}}$, Catania, Italy

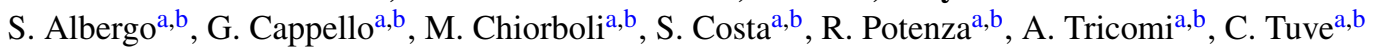

INFN Sezione di Firenze ${ }^{\mathrm{a}}$, Università di Firenze ${ }^{\mathrm{b}}$, Firenze, Italy

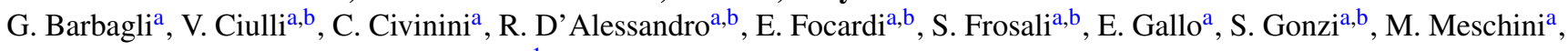
S. Paoletti ${ }^{\mathrm{a}}$, G. Sguazzoni ${ }^{\mathrm{a}}$, A. Tropiano ${ }^{\mathrm{a}, 1}$

INFN Laboratori Nazionali di Frascati, Frascati, Italy

L. Benussi, S. Bianco, S. Colafranceschi ${ }^{24}$, F. Fabbri, D. Piccolo

INFN Sezione di Genova, Genova, Italy

P. Fabbricatore, R. Musenich

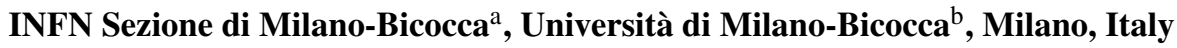

A. Benagliaa,b,1, F. De Guio ${ }^{\mathrm{a}, \mathrm{b}}$, L. Di Matteo ${ }^{\mathrm{a}, \mathrm{b}}$, S. Fiorendi ${ }^{\mathrm{a}, \mathrm{b}}$, S. Gennai ${ }^{\mathrm{a}, 1}$, A. Ghezzi ${ }^{\mathrm{a}, \mathrm{b}}$, S. Malvezzi ${ }^{\mathrm{a}}$, R.A. Manzoni ${ }^{\mathrm{a}, \mathrm{b}}$, A. Martelli ${ }^{\mathrm{a}, \mathrm{b}}$, A. Massironi ${ }^{\mathrm{a}, \mathrm{b}, 1}$, D. Menasce ${ }^{\mathrm{a}}$, L. Moroni ${ }^{\mathrm{a}}$, M. Paganoni ${ }^{\mathrm{a}, \mathrm{b}}$, D. Pedrini ${ }^{\mathrm{a}}$, S. Ragazzi ${ }^{\mathrm{a}, \mathrm{b}}$, N. Redaelli $^{\mathrm{a}}$, S. Sala $^{\mathrm{a}}$, T. Tabarelli de Fatis ${ }^{\mathrm{a}, \mathrm{b}}$

INFN Sezione di Napolia, Università di Napoli "Federico II"b, Napoli, Italy

S. Buontempo ${ }^{\mathrm{a}}$, C.A. Carrillo Montoya ${ }^{\mathrm{a}, 1}$, N. Cavallo ${ }^{\mathrm{a}, 25}$, A. De Cosa ${ }^{\mathrm{a}, \mathrm{b}}$, O. Dogangun ${ }^{\mathrm{a}, \mathrm{b}}$, F. Fabozzi $\mathrm{j}^{\mathrm{a}, 25}$, A.O.M. Iorio ${ }^{\mathrm{a}, 1}$, L. Lista ${ }^{\mathrm{a}}$, M. Merola ${ }^{\mathrm{a}, \mathrm{b}}$, P. Paolucci ${ }^{\mathrm{a}}$

INFN Sezione di Padova ${ }^{\mathrm{a}}$, Università di Padova ${ }^{\mathrm{b}}$, Università di Trento (Trento) ${ }^{\mathrm{c}}$, Padova, Italy

P. Azzi ${ }^{\mathrm{a}}$, N. Bacchetta ${ }^{\mathrm{a}, 1}$, P. Bellan ${ }^{\mathrm{a}, \mathrm{b}}$, D. Bisello ${ }^{\mathrm{a}, \mathrm{b}}$, A. Branca $^{\mathrm{a}}$, R. Carlinn, ${ }^{\mathrm{a}, \mathrm{b}}$, Phecchia ${ }^{\mathrm{a}}$, T. Dorigo ${ }^{\mathrm{a}}$, U. Dosselli ${ }^{\mathrm{a}}$, F. Gasparini ${ }^{\mathrm{a}, \mathrm{b}}$, U. Gasparini ${ }^{\mathrm{a}, \mathrm{b}}$, A. Gozzelino ${ }^{\mathrm{a}}$, K. Kanishcheve ${ }^{\mathrm{a}, \mathrm{c}}$, S. Lacaprara ${ }^{\mathrm{a}, 26}$, I. Lazzizzera ${ }^{\mathrm{a}, \mathrm{c}}$, M. Margonia ${ }^{\mathrm{a}, \mathrm{b}}$, M. Mazzucato ${ }^{\mathrm{a}}$, A.T. Meneguzzo ${ }^{\mathrm{a}, \mathrm{b}}$, M. Nespolo ${ }^{\mathrm{a}, 1}$, L. Perrozzi ${ }^{\mathrm{a}}$, N. Pozzobon ${ }^{\mathrm{a}, \mathrm{b}}$, P. Ronchese ${ }^{\mathrm{a}, \mathrm{b}}$, F. Simonetto ${ }^{\mathrm{a}, \mathrm{b}}$,

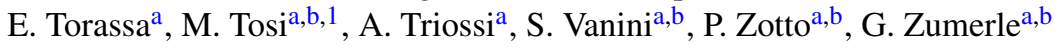

INFN Sezione di Pavia ${ }^{\mathrm{a}}$, Università di Pavia ${ }^{\mathrm{b}}$, Pavia, Italy

U. Berzano ${ }^{\mathrm{a}}$, M. Gabusi ${ }^{\mathrm{a}, \mathrm{b}}$, S.P. Rattia ${ }^{\mathrm{a}, \mathrm{b}}$, C. Riccardia ${ }^{\mathrm{a}, \mathrm{b}}$, P. Torre ${ }^{\mathrm{a}, \mathrm{b}}$, P. Vitulo ${ }^{\mathrm{a}, \mathrm{b}}$

INFN Sezione di Perugia ${ }^{\mathrm{a}}$, Università di Perugia ${ }^{\mathrm{b}}$, Perugia, Italy

M. Biasini ${ }^{\mathrm{a}, \mathrm{b}}$, G.M. Bilei ${ }^{\mathrm{a}}$, B. Caponeri ${ }^{\mathrm{a}, \mathrm{b}}$, L. Fanò ${ }^{\mathrm{a}, \mathrm{b}}$, P. Lariccia ${ }^{\mathrm{a}, \mathrm{b}}$, A. Lucaroni ${ }^{\mathrm{a}, \mathrm{b}, 1}$, G. Mantovani ${ }^{\mathrm{a}, \mathrm{b}}$, M. Menichelli ${ }^{\mathrm{a}}$, A. Nappi ${ }^{\mathrm{a}, \mathrm{b}}$, F. Romeo ${ }^{\mathrm{a}, \mathrm{b}}$, A. Santocchia ${ }^{\mathrm{a}, \mathrm{b}}$, S. Taroni ${ }^{\mathrm{a}, \mathrm{b}, 1}$, M. Valdata ${ }^{\mathrm{a}, \mathrm{b}}$

INFN Sezione di Pisa ${ }^{\mathrm{a}}$, Università di Pisa ${ }^{\mathrm{b}}$, Scuola Normale Superiore di Pisa ${ }^{\mathrm{c}}$, Pisa, Italy

P. Azzurri ${ }^{\mathrm{a}, \mathrm{c}}$, G. Bagliesi ${ }^{\mathrm{a}}$, T. Boccali ${ }^{\mathrm{a}}$, G. Broccolo ${ }^{\mathrm{a}, \mathrm{c}}$, R. Castaldi $^{\mathrm{a}}$, R.T. D’Agnolo ${ }^{\mathrm{a}, \mathrm{c}}$, R. Dell'Orso ${ }^{\mathrm{a}}$, F. Fiori ${ }^{\mathrm{a}, \mathrm{b}}$, L. Foà ${ }^{\mathrm{a}, \mathrm{c}}$,

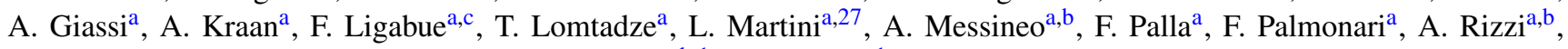

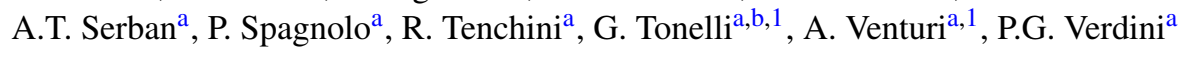

INFN Sezione di Roma ${ }^{\text {a }}$, Università di Roma "La Sapienza", , Roma, Italy

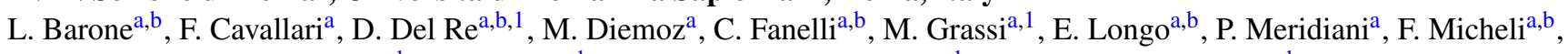
S. Nourbakhsh ${ }^{\mathrm{a}}$, G. Organtini ${ }^{\mathrm{a}, \mathrm{b}}$, F. Pandolfi ${ }^{\mathrm{a}, \mathrm{b}}$, R. Paramatti ${ }^{\mathrm{a}}$, S. Rahatlou ${ }^{\mathrm{a}, \mathrm{b}}$, M. Sigamani ${ }^{\mathrm{a}}$, L. Soffi ${ }^{\mathrm{a}, \mathrm{b}}$

INFN Sezione di Torino ${ }^{\mathrm{a}}$, Università di Torino ${ }^{\mathrm{b}}$, Università del Piemonte Orientale (Novara ${ }^{\mathrm{c}}$, Torino, Italy N. Amapane ${ }^{\mathrm{a}, \mathrm{b}}$, R. Arcidiacono ${ }^{\mathrm{a}, \mathrm{c}}$, S. Argiro ${ }^{\mathrm{a}, \mathrm{b}}$, M. Arneodo ${ }^{\mathrm{a}, \mathrm{c}}$, C. Biino ${ }^{\mathrm{a}}$, C. Botta ${ }^{\mathrm{a}, \mathrm{b}}$, N. Cartiglia ${ }^{\mathrm{a}}$, R. Castello ${ }^{\mathrm{a}, \mathrm{b}}$, M. Costa ${ }^{\mathrm{a}, \mathrm{b}}$, N. Demaria ${ }^{\mathrm{a}}$, A. Graziano ${ }^{\mathrm{a}, \mathrm{b}}$, C. Mariotti ${ }^{\mathrm{a}, 1}$, S. Maselli ${ }^{\mathrm{a}}$, E. Migliore ${ }^{\mathrm{a}, \mathrm{b}}$, V. Monaco ${ }^{\mathrm{a}, \mathrm{b}}$, M. Musich ${ }^{\mathrm{a}}$, M.M. Obertino ${ }^{\mathrm{a}, \mathrm{c}}$, N. Pastrone ${ }^{\mathrm{a}}$, M. Pelliccioni ${ }^{\mathrm{a}}$, A. Potenza ${ }^{\mathrm{a}, \mathrm{b}}$, A. Romero ${ }^{\mathrm{a}, \mathrm{b}}$, M. Ruspa ${ }^{\mathrm{a}, \mathrm{c}}$, R. Sacchi ${ }^{\mathrm{a}, \mathrm{b}}$, V. Sola ${ }^{\mathrm{a}, \mathrm{b}}$, A. Solano ${ }^{\mathrm{a}, \mathrm{b}}$, A. Staiano ${ }^{\mathrm{a}}$, A. Vilela Pereira ${ }^{\mathrm{a}}$

INFN Sezione di Trieste ${ }^{\mathrm{a}}$, Università di Trieste ${ }^{\mathrm{b}}$, Trieste, Italy

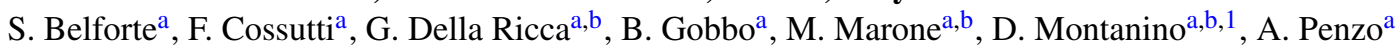

Kangwon National University, Chunchon, Korea

S.G. Heo, S.K. Nam 
Kyungpook National University, Daegu, Korea

S. Chang, J. Chung, D.H. Kim, G.N. Kim, J.E. Kim, D.J. Kong, H. Park, S.R. Ro, D.C. Son

Chonnam National University, Institute for Universe and Elementary Particles, Kwangju, Korea

J.Y. Kim, Z.J. Kim, S. Song

Konkuk University, Seoul, Korea

H.Y. Jo

Korea University, Seoul, Korea

S. Choi, D. Gyun, B. Hong, M. Jo, H. Kim, T.J. Kim, K.S. Lee, D.H. Moon, S.K. Park, E. Seo, K.S. Sim

University of Seoul, Seoul, Korea

M. Choi, S. Kang, H. Kim, J.H. Kim, C. Park, I.C. Park, S. Park, G. Ryu

Sungkyunkwan University, Suwon, Korea

Y. Cho, Y. Choi, Y.K. Choi, J. Goh, M.S. Kim, B. Lee, J. Lee, S. Lee, H. Seo, I. Yu

Vilnius University, Vilnius, Lithuania

M.J. Bilinskas, I. Grigelionis, M. Janulis

Centro de Investigacion y de Estudios Avanzados del IPN, Mexico City, Mexico

H. Castilla-Valdez, E. De La Cruz-Burelo, I. Heredia-de La Cruz, R. Lopez-Fernandez, R. Magaña Villalba, J. MartínezOrtega, A. Sánchez-Hernández, L.M. Villasenor-Cendejas

Universidad Iberoamericana, Mexico City, Mexico

S. Carrillo Moreno, F. Vazquez Valencia

Benemerita Universidad Autonoma de Puebla, Puebla, Mexico

H.A. Salazar Ibarguen

Universidad Autónoma de San Luis Potosí, San Luis Potosí, Mexico

E. Casimiro Linares, A. Morelos Pineda, M.A. Reyes-Santos

University of Auckland, Auckland, New Zealand

D. Krofcheck

University of Canterbury, Christchurch, New Zealand

A.J. Bell, P.H. Butler, R. Doesburg, S. Reucroft, H. Silverwood

National Centre for Physics, Quaid-I-Azam University, Islamabad, Pakistan

M. Ahmad, M.I. Asghar, H.R. Hoorani, S. Khalid, W.A. Khan, T. Khurshid, S. Qazi, M.A. Shah, M. Shoaib

Institute of Experimental Physics, Faculty of Physics, University of Warsaw, Warsaw, Poland

G. Brona, M. Cwiok, W. Dominik, K. Doroba, A. Kalinowski, M. Konecki, J. Krolikowski

\section{Soltan Institute for Nuclear Studies, Warsaw, Poland}

H. Bialkowska, B. Boimska, T. Frueboes, R. Gokieli, M. Górski, M. Kazana, K. Nawrocki, K. Romanowska-Rybinska, M. Szleper, G. Wrochna, P. Zalewski

Laboratório de Instrumentação e Física Experimental de Partículas, Lisboa, Portugal

N. Almeida, P. Bargassa, A. David, P. Faccioli, P.G. Ferreira Parracho, M. Gallinaro, P. Musella, A. Nayak, J. Pela ${ }^{1}$, P.Q. Ribeiro, J. Seixas, J. Varela, P. Vischia

Joint Institute for Nuclear Research, Dubna, Russia

S. Afanasiev, I. Belotelov, P. Bunin, M. Gavrilenko, I. Golutvin, I. Gorbunov, V. Karjavin, V. Konoplyanikov, G. Kozlov, A. Lanev, P. Moisenz, V. Palichik, V. Perelygin, S. Shmatov, V. Smirnov, A. Volodko, A. Zarubin

Petersburg Nuclear Physics Institute, Gatchina (St Petersburg), Russia

S. Evstyukhin, V. Golovtsov, Y. Ivanov, V. Kim, P. Levchenko, V. Murzin, V. Oreshkin, I. Smirnov, V. Sulimov, L. Uvarov, S. Vavilov, A. Vorobyev, An. Vorobyev 


\section{Institute for Nuclear Research, Moscow, Russia}

Yu. Andreev, A. Dermenev, S. Gninenko, N. Golubev, M. Kirsanov, N. Krasnikov, V. Matveev, G. Pivovarov, A. Toropin, S. Troitsky

\section{Institute for Theoretical and Experimental Physics, Moscow, Russia}

V. Epshteyn, M. Erofeeva, V. Gavrilov, M. Kossov ${ }^{1}$, A. Krokhotin, N. Lychkovskaya, V. Popov, G. Safronov, S. Semenov, V. Stolin, E. Vlasov, A. Zhokin

\section{Moscow State University, Moscow, Russia}

A. Belyaev, E. Boos, M. Dubinin ${ }^{4}$, L. Dudko, A. Ershov, L. Khein, O. Kodolova, I. Lokhtin, A. Markina, S. Obraztsov, M. Perfilov, A. Proskuryakov, L. Sarycheva ${ }^{\dagger}$, V. Savrin, A. Snigirev

\section{P.N. Lebedev Physical Institute, Moscow, Russia}

V. Andreev, M. Azarkin, I. Dremin, M. Kirakosyan, A. Leonidov, G. Mesyats, S.V. Rusakov, A. Vinogradov

State Research Center of Russian Federation, Institute for High Energy Physics, Protvino, Russia

I. Azhgirey, I. Bayshev, S. Bitioukov, V. Grishin ${ }^{1}$, V. Kachanov, D. Konstantinov, A. Korablev, V. Krychkine, V. Petrov, R. Ryutin, A. Sobol, L. Tourtchanovitch, S. Troshin, N. Tyurin, A. Uzunian, A. Volkov

University of Belgrade, Faculty of Physics and Vinca Institute of Nuclear Sciences, Belgrade, Serbia

P. Adzic ${ }^{28}$, M. Djordjevic, M. Ekmedzic, D. Krpic ${ }^{28}$, J. Milosevic

Centro de Investigaciones Energéticas Medioambientales y Tecnológicas (CIEMAT), Madrid, Spain

M. Aguilar-Benitez, J. Alcaraz Maestre, P. Arce, C. Battilana, E. Calvo, M. Cerrada, M. Chamizo Llatas, N. Colino, B. De La Cruz, A. Delgado Peris, C. Diez Pardos, D. Domínguez Vázquez, C. Fernandez Bedoya, J.P. Fernández Ramos, A. Ferrando, J. Flix, M.C. Fouz, P. Garcia-Abia, O. Gonzalez Lopez, S. Goy Lopez, J.M. Hernandez, M.I. Josa, G. Merino, J. Puerta Pelayo, I. Redondo, L. Romero, J. Santaolalla, M.S. Soares, C. Willmott

\section{Universidad Autónoma de Madrid, Madrid, Spain}

C. Albajar, G. Codispoti, J.F. de Trocóniz

\section{Universidad de Oviedo, Oviedo, Spain}

J. Cuevas, J. Fernandez Menendez, S. Folgueras, I. Gonzalez Caballero, L. Lloret Iglesias, J. Piedra Gomez ${ }^{29}$, J.M. Vizan Garcia

\section{Instituto de Física de Cantabria (IFCA), CSIC-Universidad de Cantabria, Santander, Spain}

J.A. Brochero Cifuentes, I.J. Cabrillo, A. Calderon, S.H. Chuang, J. Duarte Campderros, M. Felcini ${ }^{30}$, M. Fernandez, G. Gomez, J. Gonzalez Sanchez, C. Jorda, P. Lobelle Pardo, A. Lopez Virto, J. Marco, R. Marco, C. Martinez Rivero, F. Matorras, F.J. Munoz Sanchez, T. Rodrigo, A.Y. Rodríguez-Marrero, A. Ruiz-Jimeno, L. Scodellaro, M. Sobron Sanudo, I. Vila, R. Vilar Cortabitarte

\section{CERN, European Organization for Nuclear Research, Geneva, Switzerland}

D. Abbaneo, E. Auffray, G. Auzinger, P. Baillon, A.H. Ball, D. Barney, C. Bernet ${ }^{5}$, W. Bialas, G. Bianchi, P. Bloch, A. Bocci, H. Breuker, K. Bunkowski, T. Camporesi, G. Cerminara, T. Christiansen, J.A. Coarasa Perez, B. Curé, D. D’Enterria, A. De Roeck, S. Di Guida, M. Dobson, N. Dupont-Sagorin, A. Elliott-Peisert, B. Frisch, W. Funk, A. Gaddi, G. Georgiou, H. Gerwig, M. Giffels, D. Gigi, K. Gill, D. Giordano, M. Giunta, F. Glege, R. Gomez-Reino Garrido, P. Govoni, S. Gowdy, R. Guida, L. Guiducci, M. Hansen, P. Harris, C. Hartl, J. Harvey, B. Hegner, A. Hinzmann, H.F. Hoffmann, V. Innocente, P. Janot, K. Kaadze, E. Karavakis, K. Kousouris, P. Lecoq, P. Lenzi, C. Lourenço, T. Mäki, M. Malberti, L. Malgeri, M. Mannelli, L. Masetti, G. Mavromanolakis, F. Meijers, S. Mersi, E. Meschi, R. Moser, M.U. Mozer, M. Mulders, E. Nesvold, M. Nguyen, T. Orimoto, L. Orsini, E. Palencia Cortezon, E. Perez, A. Petrilli, A. Pfeiffer, M. Pierini, M. Pimiä, D. Piparo, G. Polese, L. Quertenmont, A. Racz, W. Reece, J. Rodrigues Antunes, G. Rolandi ${ }^{31}$, T. Rommerskirchen, C. Rovelli ${ }^{32}$, M. Rovere, H. Sakulin, F. Santanastasio, C. Schäfer, C. Schwick, I. Segoni, A. Sharma, P. Siegrist, P. Silva, M. Simon, P. Sphicas ${ }^{33}$, D. Spiga, M. Spiropulu ${ }^{4}$, M. Stoye, A. Tsirou, G.I. Veres ${ }^{16}$, P. Vichoudis, H.K. Wöhri, S.D. Worm ${ }^{34}$, W.D. Zeuner

\section{Paul Scherrer Institut, Villigen, Switzerland}

W. Bertl, K. Deiters, W. Erdmann, K. Gabathuler, R. Horisberger, Q. Ingram, H.C. Kaestli, S. König, D. Kotlinski, U. Langenegger, F. Meier, D. Renker, T. Rohe, J. Sibille ${ }^{35}$ 
Institute for Particle Physics, ETH Zurich, Zurich, Switzerland

L. Bäni, P. Bortignon, M.A. Buchmann, B. Casal, N. Chanon, Z. Chen, A. Deisher, G. Dissertori, M. Dittmar, M. Dünser, J. Eugster, K. Freudenreich, C. Grab, P. Lecomte, W. Lustermann, P. Martinez Ruiz del Arbol, N. Mohr, F. Moortgat, C. Nägeli ${ }^{36}$, P. Nef, F. Nessi-Tedaldi, L. Pape, F. Pauss, M. Peruzzi, F.J. Ronga, M. Rossini, L. Sala, A.K. Sanchez, M.C. Sawley, A. Starodumov ${ }^{37}$, B. Stieger, M. Takahashi, L. Tauscher ${ }^{\dagger}$, A. Thea, K. Theofilatos, D. Treille, C. Urscheler, R. Wallny, H.A. Weber, L. Wehrli, J. Weng

Universität Zürich, Zurich, Switzerland

E. Aguilo, C. Amsler, V. Chiochia, S. De Visscher, C. Favaro, M. Ivova Rikova, B. Millan Mejias, P. Otiougova, P. Robmann, H. Snoek, M. Verzetti

\section{National Central University, Chung-Li, Taiwan}

Y.H. Chang, K.H. Chen, C.M. Kuo, S.W. Li, W. Lin, Z.K. Liu, Y.J. Lu, D. Mekterovic, R. Volpe, S.S. Yu

\section{National Taiwan University (NTU), Taipei, Taiwan}

P. Bartalini, P. Chang, Y.H. Chang, Y.W. Chang, Y. Chao, K.F. Chen, C. Dietz, U. Grundler, W.-S. Hou, Y. Hsiung, K.Y. Kao, Y.J. Lei, R.-S. Lu, D. Majumder, E. Petrakou, X. Shi, J.G. Shiu, Y.M. Tzeng, M. Wang

\section{Cukurova University, Adana, Turkey}

A. Adiguzel, M.N. Bakirci ${ }^{38}$, S. Cerci ${ }^{39}$, C. Dozen, I. Dumanoglu, E. Eskut, S. Girgis, G. Gokbulut, I. Hos, E.E. Kangal, G. Karapinar, A. Kayis Topaksu, G. Onengut, K. Ozdemir, S. Ozturk ${ }^{40}$, A. Polatoz, K. Sogut ${ }^{41}$, D. Sunar Cerci ${ }^{39}$, B. Tali ${ }^{39}$, H. Topakli ${ }^{38}$, D. Uzun, L.N. Vergili, M. Vergili

Middle East Technical University, Physics Department, Ankara, Turkey

I.V. Akin, T. Aliev, B. Bilin, S. Bilmis, M. Deniz, H. Gamsizkan, A.M. Guler, K. Ocalan, A. Ozpineci, M. Serin, R. Sever, U.E. Surat, M. Yalvac, E. Yildirim, M. Zeyrek

\section{Bogazici University, Istanbul, Turkey}

M. Deliomeroglu, E. Gülmez, B. Isildak, M. Kaya ${ }^{42}$, O. Kaya ${ }^{42}$, S. Ozkorucuklu ${ }^{43}$, N. Sonmez ${ }^{44}$

\section{National Scientific Center, Kharkov Institute of Physics and Technology, Kharkov, Ukraine}

L. Levchuk

\section{University of Bristol, Bristol, United Kingdom}

F. Bostock, J.J. Brooke, E. Clement, D. Cussans, H. Flacher, R. Frazier, J. Goldstein, M. Grimes, G.P. Heath, H.F. Heath, L. Kreczko, S. Metson, D.M. Newbold ${ }^{34}$, K. Nirunpong, A. Poll, S. Senkin, V.J. Smith, T. Williams

\section{Rutherford Appleton Laboratory, Didcot, United Kingdom}

L. Basso ${ }^{45}$, K.W. Bell, A. Belyaev ${ }^{45}$, C. Brew, R.M. Brown, D.J.A. Cockerill, J.A. Coughlan, K. Harder, S. Harper, J. Jackson, B.W. Kennedy, E. Olaiya, D. Petyt, B.C. Radburn-Smith, C.H. Shepherd-Themistocleous, I.R. Tomalin, W.J. Womersley

\section{Imperial College, London, United Kingdom}

R. Bainbridge, G. Ball, R. Beuselinck, O. Buchmuller, D. Colling, N. Cripps, M. Cutajar, P. Dauncey, G. Davies, M. Della Negra, W. Ferguson, J. Fulcher, D. Futyan, A. Gilbert, A. Guneratne Bryer, G. Hall, Z. Hatherell, J. Hays, G. Iles, M. Jarvis, G. Karapostoli, L. Lyons, A.-M. Magnan, J. Marrouche, B. Mathias, R. Nandi, J. Nash, A. Nikitenko ${ }^{37}$, A. Papageorgiou, M. Pesaresi, K. Petridis, M. Pioppi ${ }^{46}$, D.M. Raymond, S. Rogerson, N. Rompotis, A. Rose, M.J. Ryan, C. Seez, A. Sparrow, A. Tapper, S. Tourneur, M. Vazquez Acosta, T. Virdee, S. Wakefield, N. Wardle, D. Wardrope, T. Whyntie

\section{Brunel University, Uxbridge, United Kingdom}

M. Barrett, M. Chadwick, J.E. Cole, P.R. Hobson, A. Khan, P. Kyberd, D. Leslie, W. Martin, I.D. Reid, P. Symonds, L. Teodorescu, M. Turner

Baylor University, Waco, USA

K. Hatakeyama, H. Liu, T. Scarborough

The University of Alabama, Tuscaloosa, USA

C. Henderson

Boston University, Boston, USA

A. Avetisyan, T. Bose, E. Carrera Jarrin, C. Fantasia, A. Heister, J.St. John, P. Lawson, D. Lazic, J. Rohlf, D. Sperka, L. Sulak 


\section{Brown University, Providence, USA}

S. Bhattacharya, D. Cutts, A. Ferapontov, U. Heintz, S. Jabeen, G. Kukartsev, G. Landsberg, M. Luk, M. Narain, D. Nguyen, M. Segala, T. Sinthuprasith, T. Speer, K.V. Tsang

\section{University of California, Davis, Davis, USA}

R. Breedon, G. Breto, M. Calderon De La Barca Sanchez, M. Caulfield, S. Chauhan, M. Chertok, J. Conway, R. Conway, P.T. Cox, J. Dolen, R. Erbacher, M. Gardner, R. Houtz, W. Ko, A. Kopecky, R. Lander, O. Mall, T. Miceli, R. Nelson, D. Pellett, J. Robles, B. Rutherford, M. Searle, J. Smith, M. Squires, M. Tripathi, R. Vasquez Sierra

\section{University of California, Los Angeles, Los Angeles, USA}

V. Andreev, K. Arisaka, D. Cline, R. Cousins, J. Duris, S. Erhan, P. Everaerts, C. Farrell, J. Hauser, M. Ignatenko, C. Jarvis, C. Plager, G. Rakness, P. Schlein ${ }^{\dagger}$, J. Tucker, V. Valuev, M. Weber

\section{University of California, Riverside, Riverside, USA}

J. Babb, R. Clare, J. Ellison, J.W. Gary, F. Giordano, G. Hanson, G.Y. Jeng ${ }^{47}$, H. Liu, O.R. Long, A. Luthra, H. Nguyen, S. Paramesvaran, J. Sturdy, S. Sumowidagdo, R. Wilken, S. Wimpenny

\section{University of California, San Diego, La Jolla, USA}

W. Andrews, J.G. Branson, G.B. Cerati, S. Cittolin, D. Evans, F. Golf, A. Holzner, R. Kelley, M. Lebourgeois, J. Letts, I. Macneill, B. Mangano, S. Padhi, C. Palmer, G. Petrucciani, H. Pi, M. Pieri, R. Ranieri, M. Sani, I. Sfiligoi, V. Sharma, S. Simon, E. Sudano, M. Tadel, Y. Tu, A. Vartak, S. Wasserbaech ${ }^{48}$, F. Würthwein, A. Yagil, J. Yoo

\section{University of California, Santa Barbara, Santa Barbara, USA}

D. Barge, R. Bellan, C. Campagnari, M. D’Alfonso, T. Danielson, K. Flowers, P. Geffert, J. Incandela, C. Justus, P. Kalavase, S.A. Koay, D. Kovalskyi ${ }^{1}$, V. Krutelyov, S. Lowette, N. Mccoll, V. Pavlunin, F. Rebassoo, J. Ribnik, J. Richman, R. Rossin, D. Stuart, W. To, J.R. Vlimant, C. West

\section{California Institute of Technology, Pasadena, USA}

A. Apresyan, A. Bornheim, J. Bunn, Y. Chen, E. Di Marco, J. Duarte, M. Gataullin, Y. Ma, A. Mott, H.B. Newman, C. Rogan, V. Timciuc, P. Traczyk, J. Veverka, R. Wilkinson, Y. Yang, R.Y. Zhu

\section{Carnegie Mellon University, Pittsburgh, USA}

B. Akgun, R. Carroll, T. Ferguson, Y. Iiyama, D.W. Jang, S.Y. Jun, Y.F. Liu, M. Paulini, J. Russ, H. Vogel, I. Vorobiev

\section{University of Colorado at Boulder, Boulder, USA}

J.P. Cumalat, M.E. Dinardo, B.R. Drell, C.J. Edelmaier, W.T. Ford, A. Gaz, B. Heyburn, E. Luiggi Lopez, U. Nauenberg, J.G. Smith, K. Stenson, K.A. Ulmer, S.R. Wagner, S.L. Zang

\section{Cornell University, Ithaca, USA}

L. Agostino, J. Alexander, A. Chatterjee, N. Eggert, L.K. Gibbons, B. Heltsley, W. Hopkins, A. Khukhunaishvili, B. Kreis, N. Mirman, G. Nicolas Kaufman, J.R. Patterson, A. Ryd, E. Salvati, W. Sun, W.D. Teo, J. Thom, J. Thompson, J. Vaughan, Y. Weng, L. Winstrom, P. Wittich

\section{Fairfield University, Fairfield, USA}

A. Biselli, D. Winn

\section{Fermi National Accelerator Laboratory, Batavia, USA}

S. Abdullin, M. Albrow, J. Anderson, G. Apollinari, M. Atac, J.A. Bakken, L.A.T. Bauerdick, A. Beretvas, J. Berryhill, P.C. Bhat, I. Bloch, K. Burkett, J.N. Butler, V. Chetluru, H.W.K. Cheung, F. Chlebana, S. Cihangir, W. Cooper, D.P. Eartly, V.D. Elvira, S. Esen, I. Fisk, J. Freeman, Y. Gao, E. Gottschalk, D. Green, O. Gutsche, J. Hanlon, R.M. Harris, J. Hirschauer, B. Hooberman, H. Jensen, S. Jindariani, M. Johnson, U. Joshi, B. Klima, S. Kunori, S. Kwan, C. Leonidopoulos, D. Lincoln, R. Lipton, J. Lykken, K. Maeshima, J.M. Marraffino, S. Maruyama, D. Mason, P. McBride, T. Miao, K. Mishra, S. Mrenna, Y. Musienko ${ }^{49}$, C. Newman-Holmes, V. O’Dell, J. Pivarski, R. Pordes, O. Prokofyev, T. Schwarz, E. Sexton-Kennedy, S. Sharma, W.J. Spalding, L. Spiegel, P. Tan, L. Taylor, S. Tkaczyk, L. Uplegger, E.W. Vaandering, R. Vidal, J. Whitmore, W. Wu, F. Yang, F. Yumiceva, J.C. Yun

\section{University of Florida, Gainesville, USA}

D. Acosta, P. Avery, D. Bourilkov, M. Chen, S. Das, M. De Gruttola, G.P. Di Giovanni, D. Dobur, A. Drozdetskiy, R.D. Field, M. Fisher, Y. Fu, I.K. Furic, J. Gartner, S. Goldberg, J. Hugon, B. Kim, J. Konigsberg, A. Korytov, A. Kropivnitskaya, T. Kypreos, J.F. Low, K. Matchev, P. Milenovic ${ }^{50}$, G. Mitselmakher, L. Muniz, R. Remington, A. Rinkevicius, M. Schmitt, B. Scurlock, P. Sellers, N. Skhirtladze, M. Snowball, D. Wang, J. Yelton, M. Zakaria 
Florida International University, Miami, USA

V. Gaultney, L.M. Lebolo, S. Linn, P. Markowitz, G. Martinez, J.L. Rodriguez

Florida State University, Tallahassee, USA

T. Adams, A. Askew, J. Bochenek, J. Chen, B. Diamond, S.V. Gleyzer, J. Haas, S. Hagopian, V. Hagopian, M. Jenkins, K.F. Johnson, H. Prosper, S. Sekmen, V. Veeraraghavan, M. Weinberg

Florida Institute of Technology, Melbourne, USA

M.M. Baarmand, B. Dorney, M. Hohlmann, H. Kalakhety, I. Vodopiyanov

University of Illinois at Chicago (UIC), Chicago, USA

M.R. Adams, I.M. Anghel, L. Apanasevich, Y. Bai, V.E. Bazterra, R.R. Betts, J. Callner, R. Cavanaugh, C. Dragoiu, L. Gauthier, C.E. Gerber, D.J. Hofman, S. Khalatyan, G.J. Kunde ${ }^{51}$, F. Lacroix, M. Malek, C. O'Brien, C. Silkworth, C. Silvestre, D. Strom, N. Varelas

The University of Iowa, Iowa City, USA

U. Akgun, E.A. Albayrak, B. Bilki ${ }^{52}$, W. Clarida, F. Duru, S. Griffiths, C.K. Lae, E. McCliment, J.-P. Merlo, H. Mermerkaya ${ }^{53}$, A. Mestvirishvili, A. Moeller, J. Nachtman, C.R. Newsom, E. Norbeck, J. Olson, Y. Onel, F. Ozok, S. Sen, E. Tiras, J. Wetzel, T. Yetkin, K. Yi

Johns Hopkins University, Baltimore, USA

B.A. Barnett, B. Blumenfeld, S. Bolognesi, A. Bonato, D. Fehling, G. Giurgiu, A.V. Gritsan, Z.J. Guo, G. Hu, P. Maksimovic, S. Rappoccio, M. Swartz, N.V. Tran, A. Whitbeck

The University of Kansas, Lawrence, USA

P. Baringer, A. Bean, G. Benelli, O. Grachov, R.P. Kenny Iii, M. Murray, D. Noonan, S. Sanders, R. Stringer, G. Tinti, J.S. Wood, V. Zhukova

Kansas State University, Manhattan, USA

A.F. Barfuss, T. Bolton, I. Chakaberia, A. Ivanov, S. Khalil, M. Makouski, Y. Maravin, S. Shrestha, I. Svintradze

Lawrence Livermore National Laboratory, Livermore, USA

J. Gronberg, D. Lange, D. Wright

University of Maryland, College Park, USA

A. Baden, M. Boutemeur, B. Calvert, S.C. Eno, J.A. Gomez, N.J. Hadley, R.G. Kellogg, M. Kirn, T. Kolberg, Y. Lu, M. Marionneau, A.C. Mignerey, A. Peterman, K. Rossato, P. Rumerio, A. Skuja, J. Temple, M.B. Tonjes, S.C. Tonwar, E. Twedt

\section{Massachusetts Institute of Technology, Cambridge, USA}

B. Alver, G. Bauer, J. Bendavid, W. Busza, E. Butz, I.A. Cali, M. Chan, V. Dutta, G. Gomez Ceballos, M. Goncharov, K.A. Hahn, Y. Kim, M. Klute, Y.-J. Lee, W. Li, P.D. Luckey, T. Ma, S. Nahn, C. Paus, D. Ralph, C. Roland, G. Roland, M. Rudolph, G.S.F. Stephans, F. Stöckli, K. Sumorok, K. Sung, D. Velicanu, E.A. Wenger, R. Wolf, B. Wyslouch, S. Xie, M. Yang, Y. Yilmaz, A.S. Yoon, M. Zanetti

University of Minnesota, Minneapolis, USA

S.I. Cooper, P. Cushman, B. Dahmes, A. De Benedetti, G. Franzoni, A. Gude, J. Haupt, S.C. Kao, K. Klapoetke, Y. Kubota, J. Mans, N. Pastika, V. Rekovic, R. Rusack, M. Sasseville, A. Singovsky, N. Tambe, J. Turkewitz

University of Mississippi, University, USA

L.M. Cremaldi, R. Godang, R. Kroeger, L. Perera, R. Rahmat, D.A. Sanders, D. Summers

University of Nebraska-Lincoln, Lincoln, USA

E. Avdeeva, K. Bloom, S. Bose, J. Butt, D.R. Claes, A. Dominguez, M. Eads, P. Jindal, J. Keller, I. Kravchenko, J. LazoFlores, H. Malbouisson, S. Malik, G.R. Snow

State University of New York at Buffalo, Buffalo, USA

U. Baur, A. Godshalk, I. Iashvili, S. Jain, A. Kharchilava, A. Kumar, S.P. Shipkowski, K. Smith, Z. Wan

Northeastern University, Boston, USA

G. Alverson, E. Barberis, D. Baumgartel, M. Chasco, D. Trocino, D. Wood, J. Zhang 
Northwestern University, Evanston, USA

A. Anastassov, A. Kubik, N. Mucia, N. Odell, R.A. Ofierzynski, B. Pollack, A. Pozdnyakov, M. Schmitt, S. Stoynev, M. Velasco, S. Won

\section{University of Notre Dame, Notre Dame, USA}

L. Antonelli, D. Berry, A. Brinkerhoff, M. Hildreth, C. Jessop, D.J. Karmgard, J. Kolb, K. Lannon, W. Luo, S. Lynch, N. Marinelli, D.M. Morse, T. Pearson, R. Ruchti, J. Slaunwhite, N. Valls, M. Wayne, M. Wolf, J. Ziegler

The Ohio State University, Columbus, USA

B. Bylsma, L.S. Durkin, C. Hill, P. Killewald, K. Kotov, T.Y. Ling, D. Puigh, M. Rodenburg, C. Vuosalo, G. Williams

\section{Princeton University, Princeton, USA}

N. Adam, E. Berry, P. Elmer, D. Gerbaudo, V. Halyo, P. Hebda, J. Hegeman, A. Hunt, E. Laird, D. Lopes Pegna, P. Lujan, D. Marlow, T. Medvedeva, M. Mooney, J. Olsen, P. Piroué, X. Quan, A. Raval, H. Saka, D. Stickland, C. Tully, J.S. Werner, A. Zuranski

University of Puerto Rico, Mayaguez, USA

J.G. Acosta, X.T. Huang, A. Lopez, H. Mendez, S. Oliveros, J.E. Ramirez Vargas, A. Zatserklyaniy

Purdue University, West Lafayette, USA

E. Alagoz, V.E. Barnes, D. Benedetti, G. Bolla, D. Bortoletto, M. De Mattia, A. Everett, L. Gutay, Z. Hu, M. Jones, O. Koybasi, M. Kress, A.T. Laasanen, N. Leonardo, V. Maroussov, P. Merkel, D.H. Miller, N. Neumeister, I. Shipsey, D. Silvers, A. Svyatkovskiy, M. Vidal Marono, H.D. Yoo, J. Zablocki, Y. Zheng

\section{Purdue University Calumet, Hammond, USA}

S. Guragain, N. Parashar

\section{Rice University, Houston, USA}

A. Adair, C. Boulahouache, V. Cuplov, K.M. Ecklund, F.J.M. Geurts, B.P. Padley, R. Redjimi, J. Roberts, J. Zabel

\section{University of Rochester, Rochester, USA}

B. Betchart, A. Bodek, Y.S. Chung, R. Covarelli, P. de Barbaro, R. Demina, Y. Eshaq, A. Garcia-Bellido, P. Goldenzweig, Y. Gotra, J. Han, A. Harel, D.C. Miner, G. Petrillo, W. Sakumoto, D. Vishnevskiy, M. Zielinski

\section{The Rockefeller University, New York, USA}

A. Bhatti, R. Ciesielski, L. Demortier, K. Goulianos, G. Lungu, S. Malik, C. Mesropian

\section{Rutgers, the State University of New Jersey, Piscataway, USA}

S. Arora, O. Atramentov, A. Barker, J.P. Chou, C. Contreras-Campana, E. Contreras-Campana, D. Duggan, D. Ferencek, Y. Gershtein, R. Gray, E. Halkiadakis, D. Hidas, D. Hits, A. Lath, S. Panwalkar, M. Park, R. Patel, A. Richards, K. Rose, S. Salur, S. Schnetzer, C. Seitz, S. Somalwar, R. Stone, S. Thomas

\section{University of Tennessee, Knoxville, USA}

G. Cerizza, M. Hollingsworth, S. Spanier, Z.C. Yang, A. York

\section{Texas A\&M University, College Station, USA}

R. Eusebi, W. Flanagan, J. Gilmore, T. Kamon ${ }^{54}$, V. Khotilovich, R. Montalvo, I. Osipenkov, Y. Pakhotin, A. Perloff, J. Roe, A. Safonov, T. Sakuma, S. Sengupta, I. Suarez, A. Tatarinov, D. Toback

\section{Texas Tech University, Lubbock, USA}

N. Akchurin, C. Bardak, J. Damgov, P.R. Dudero, C. Jeong, K. Kovitanggoon, S.W. Lee, T. Libeiro, P. Mane, Y. Roh, A. Sill, I. Volobouev, R. Wigmans

\section{Vanderbilt University, Nashville, USA}

E. Appelt, E. Brownson, D. Engh, C. Florez, W. Gabella, A. Gurrola, M. Issah, W. Johns, P. Kurt, C. Maguire, A. Melo, P. Sheldon, B. Snook, S. Tuo, J. Velkovska

\section{University of Virginia, Charlottesville, USA}

M.W. Arenton, M. Balazs, S. Boutle, S. Conetti, B. Cox, B. Francis, S. Goadhouse, J. Goodell, R. Hirosky, A. Ledovskoy, C. Lin, C. Neu, J. Wood, R. Yohay 


\section{Wayne State University, Detroit, USA}

S. Gollapinni, R. Harr, P.E. Karchin, C. Kottachchi Kankanamge Don, P. Lamichhane, M. Mattson, C. Milstène, A. Sakharov

\section{University of Wisconsin, Madison, USA}

M. Anderson, M. Bachtis, D. Belknap, J.N. Bellinger, J. Bernardini, L. Borrello, D. Carlsmith, M. Cepeda, S. Dasu, J. Efron, E. Friis, L. Gray, K.S. Grogg, M. Grothe, R. Hall-Wilton, M. Herndon, A. Hervé, P. Klabbers, J. Klukas, A. Lanaro, C. Lazaridis, J. Leonard, R. Loveless, A. Mohapatra, I. Ojalvo, G.A. Pierro, I. Ross, A. Savin, W.H. Smith, J. Swanson

$\dagger$ : Deceased

1: Also at CERN, European Organization for Nuclear Research, Geneva, Switzerland

2: Also at National Institute of Chemical Physics and Biophysics, Tallinn, Estonia

3: Also at Universidade Federal do ABC, Santo Andre, Brazil

4: Also at California Institute of Technology, Pasadena, USA

5: Also at Laboratoire Leprince-Ringuet, Ecole Polytechnique, IN2P3-CNRS, Palaiseau, France

6: Also at Suez Canal University, Suez, Egypt

7: Also at Cairo University, Cairo, Egypt

8: Also at British University, Cairo, Egypt

9: Also at Fayoum University, El-Fayoum, Egypt

10: Now at Ain Shams University, Cairo, Egypt

11: Also at Soltan Institute for Nuclear Studies, Warsaw, Poland

12: Also at Université de Haute-Alsace, Mulhouse, France

13: Also at Moscow State University, Moscow, Russia

14: Also at Brandenburg University of Technology, Cottbus, Germany

15: Also at Institute of Nuclear Research ATOMKI, Debrecen, Hungary

16: Also at Eötvös Loránd University, Budapest, Hungary

17: Also at Tata Institute of Fundamental Research - HECR, Mumbai, India

18: Now at King Abdulaziz University, Jeddah, Saudi Arabia

19: Also at University of Visva-Bharati, Santiniketan, India

20: Also at Sharif University of Technology, Tehran, Iran

21: Also at Isfahan University of Technology, Isfahan, Iran

22: Also at Shiraz University, Shiraz, Iran

23: Also at Plasma Physics Research Center, Science and Research Branch, Islamic Azad University, Teheran, Iran

24: Also at Facoltà Ingegneria Università di Roma, Roma, Italy

25: Also at Università della Basilicata, Potenza, Italy

26: Also at Laboratori Nazionali di Legnaro dell' INFN, Legnaro, Italy

27: Also at Università degli studi di Siena, Siena, Italy

28: Also at Faculty of Physics of University of Belgrade, Belgrade, Serbia

29: Also at University of Florida, Gainesville, USA

30: Also at University of California, Los Angeles, Los Angeles, USA

31: Also at Scuola Normale e Sezione dell' INFN, Pisa, Italy

32: Also at INFN Sezione di Roma; Università di Roma "La Sapienza", Roma, Italy

33: Also at University of Athens, Athens, Greece

34: Also at Rutherford Appleton Laboratory, Didcot, United Kingdom

35: Also at The University of Kansas, Lawrence, USA

36: Also at Paul Scherrer Institut, Villigen, Switzerland

37: Also at Institute for Theoretical and Experimental Physics, Moscow, Russia

38: Also at Gaziosmanpasa University, Tokat, Turkey

39: Also at Adiyaman University, Adiyaman, Turkey

40: Also at The University of Iowa, Iowa City, USA

41: Also at Mersin University, Mersin, Turkey

42: Also at Kafkas University, Kars, Turkey

43: Also at Suleyman Demirel University, Isparta, Turkey

44: Also at Ege University, Izmir, Turkey

45: Also at School of Physics and Astronomy, University of Southampton, Southampton, United Kingdom 
46: Also at INFN Sezione di Perugia; Università di Perugia, Perugia, Italy

47: Also at University of Sydney, Sydney, Australia

48: Also at Utah Valley University, Orem, USA

49: Also at Institute for Nuclear Research, Moscow, Russia

50: Also at University of Belgrade, Faculty of Physics and Vinca Institute of Nuclear Sciences, Belgrade, Serbia

51: Also at Los Alamos National Laboratory, Los Alamos, USA

52: Also at Argonne National Laboratory, Argonne, USA

53: Also at Erzincan University, Erzincan, Turkey

54: Also at Kyungpook National University, Daegu, Korea 